\title{
Insight of COVID-19/ SARS-CoV-2 and its Probable Treatment - A Mathematical Approach
}

Amar Nath Chatterjee

K.L.S. COLLEGE, NAWADA https://orcid.org/0000-0002-3007-0144

\section{Shubhankar Saha}

Sir Gurudas Mahavidyalaya, Kolkata, India

Priti Kumar Roy ( $\nabla$ pritiju@gmail.com )

JADAVPUR UNIVERSITY

\section{Fahad Al Basir}

ASANSOL GIRLS' COLLEGE, WEST BENGAL, INDIA

\section{Evgenii Khailov}

Moscow State Lomonosov University, Russia

\section{Ellina Grigorieva}

Texas Woman's University, Denton TX 76204, USA

\section{Research Article}

Keywords: COVID-19, SARS-CoV-2, Hydroxychloroquine, Lipopeptide, Chaos, Impulsive differential equation

Posted Date: June 12th, 2020

DOI: https://doi.org/10.21203/rs.3.rs-34519/v1

License: (c) (i) This work is licensed under a Creative Commons Attribution 4.0 International License.

Read Full License 


\title{
Insight of COVID-19/ SARS-CoV-2 and its Probable Treatment -A Mathematical Approach
}

\author{
Amar Nath Chatterjee ${ }^{1}$, Shubhankar Saha², Priti Kumar Roy *3, \\ Fahad Al Basir ${ }^{4}$, Evgenii Khailov ${ }^{5}$, and Ellina Grigorieva ${ }^{6}$ \\ ${ }^{1}$ Department of Mathematics, K.L.S. College, Nawada, Bihar, India. \\ ${ }^{2}$ Department of Mathematics, Sir Gurudas Mahavidyalaya, Kolkata, India. \\ ${ }^{3}$ Department of Mathematics, Jadavpur University, Kolkata, India. \\ ${ }^{4}$ Department of Mathematics, Asansol Girls' College, Asansol-4, West \\ Bebgal-713304, India. \\ ${ }^{5}$ Computational Mathematics and Cybernetics, Moscow State Lomonosov \\ University, Russia. \\ ${ }^{6}$ Department of Mathematics and Computer Sciences, Texas Woman's University, \\ Denton TX 76204, USA.
}

June 12,2020

\begin{abstract}
The novel coronavirus disease (COVID19) emerged in Wuhan, China in December 2019. In a matter of weeks, the disease had spread well outside China, and now reaching countries in all parts of the globe. Its treatment and recovery are the two most primary concerns for every country. Recently, medical science has shown some studies that reveal post-infection Hydroxychloroquine (HCQ) treatment followed by lipopeptide EK1C4 could be an effective interference in prevention of the disease COVID19, spreaded by SARS-CoV-2. However, there are some side effects of these drugs, especially for aged persons, but this is yet to be explored by rescaling the drug dosage with a proper dosing time interval.

We propose a mathematical model that explains combination drug therapy on the dynamics of SARS-CoV-2/COVID19. We apply the method of impulsive differential equation in our model and it is useful for elucidating insights into regular drug dosing. Systematic approach
\end{abstract}

${ }^{*}$ Corresponding author. Email: pritiju@gmail.com 
of this combination of drug therapy allows us to gain more fruitful results.

In this model, we first investigate the chaotic nature of the system induced by SARS-CoV-2 with and without any treatment. Then we enquire how drug therapy reduces the threshold value of infection and observe its complex dynamics. We perform equilibrium analysis, local and global stability analysis and find the region of safe dosing so that there occurs no side-effects during treatment and afterwards. Our results suggest that only proper treatment enhances the stability in a SARS-CoV-2 infected system.

Keywords: COVID-19, SARS-CoV-2, Hydroxychloroquine, Lipopeptide, Chaos, Impulsive differential equation.

\section{Introduction}

On December 31, 2019, strange pneumonia cases that did not respond well to treatment were detected in Wuhan, China. These were the first reported cases of COVID-19. On January 30, 2020, WHO names this disease COVID -19 and declared Coronavirus outbreak a pandemic on February 11, 2020. The corresponding virus name is SARS-CoV-2, which was formerly known as 2019-nCoV (see Zhou et al. (2020), Menachery et al. (2016)).

In the past two decades coronaviruses have caused two large-scale pandemics, SARS (Severe Acute Respiratory Syndrome) and MERS (Middle East respiratory syndrome)(see Zhou et al. (2020), Van et al. (2012), Rudragouda et al. (2014)). Coronaviruses cause mild respiratory illness (see Perlman S. \& Dandekar A. A. (2005), Rudragouda et al. (2014)). Usually coronavirus family, mainly SARS-CoV and MERS-CoV, cause mortal disease in the population of elder people or those of immune-compromised individuals (see Perlman S. \& Netland J(2009), Rudragouda et al. (2014)).

In 2003-2004, Severe Acute Respiratory Syndrome (SARS-CoV) caused an epidemic of SARS affected 26 countries and resulted in more than 8000 cases. At the end of the epidemic in June 2003, 8439 people have been affected, and 812 died from SARS (see Chan et al. (2003)). Novel coronavirus (Middle East respiratory syndrome coronavirus, or MERS-CoV) was first identified in Saudi Arabia in 2012. Approximately 35\% of reported patients with MERS-CoV infection have died. Since September 2012, WHO has been notified of 2494 laboratory-confirmed cases of infection with MERS$\mathrm{CoV}$ and 858 MERS-CoV associated deaths have occurred. SARS-CoV-2, spreading to over 209 countries and infecting over four and half millions of individuals and approximately $14 \%$ of reported patients with SARS-CoV-2 
infection have died as of May 19, 2020 with a closed cases of about more than two millions people.

SARS-CoV-2 that belongs to the family of coronaviruses, named for their crown-like homotrimeric class 1 fusion spike protein (or S protein) presented on their surfaces. It can cause COVID-19, a contagious viral infection that attacks primarily our throat and lungs epithelial cells. SARS-CoV and MERS-CoV enter target cells through an endosomal pathway. The S proteins spike of SARS-CoV bind to cellular receptor angiotensin-converting enzyme 2 (ACE2) of epithelial cells (see Song et al. (2019). Xia et al. (2020)) shown that SARS-CoV-2 exhibits much higher capacity of membrane fusion than SARS-CoV. Bonding affinity of ACE2 with the S1 subunit of S protein of SARS-CoV-2 is 10 to 20 fold higher than that of the SARS-CoV (see Wrapp et al. (2020)). These studies suggest that fusion machinery of SARS-CoV-2 contributes higher infectivity and transmissibility of SARSCoV-2 compared to SARS-CoV. For MERS-CoV infection, the virus-specific primary receptor is a multifunctional cell surface protein, dipeptidyl peptidase 4 (DPP4) (see Meyerholz et al. (2016)), which is widely expressed on epithelial cells in the kidney, alveoli, small intestine, liver, and prostate, and on activated leukocytes (Widagdo et al.(2016)). MERS-CoV causes acute, highly lethal pneumonia and renal dysfunction with various clinical symptoms, including but not restricted to fever, cough, sore throat, myalgia, chest pain, diarrhea, vomiting, and abdominal pain (see Chu et al. (2005), Saad et al. (2014)).

Zhou et al. (2020) developed quantitative PCR-based methods to detect SARS-CoV-2 infections. Using these methods, they verified the respiratory tract as a principal infection site. SARS-CoV-2 infects primary human airway epithelial cells. Angiotensic converting enzyme II (ACE2) receptor of epithelial cells plays an important role in cellular entry (see Zhou et al. (2020), Wan et al. (2020)). It has been observed that ACE2 can be expressed in the oral cavity. The concentration of ACE2 receptors are higher in tongue than in buccal and gingival tissues. These findings imply that the mucosa of the oral cavity may be a potentially high-risk route of COVID-19 infection. Thus epithelial cells of the tongue are the major routes of entry for COVID-19. Zhou et al. (2020) also reported that SARS-CoV-2 spikes $\mathrm{S}$ bind with ACE2 receptor of epithelial cells with high affinity. The bonding between $S$ - spike of SARS-CoV-2 with ACE2 (see Wan et al. (2020)), results from the fusion between the viral envelope and the target cell membrane and the epithelial cells become infected. The S protein plays a major role in the induction of protective immunity during the infection of SARSCoV-2 by eliciting neutralization antibody and $\mathrm{T}$ cell responses (see Hsueh 


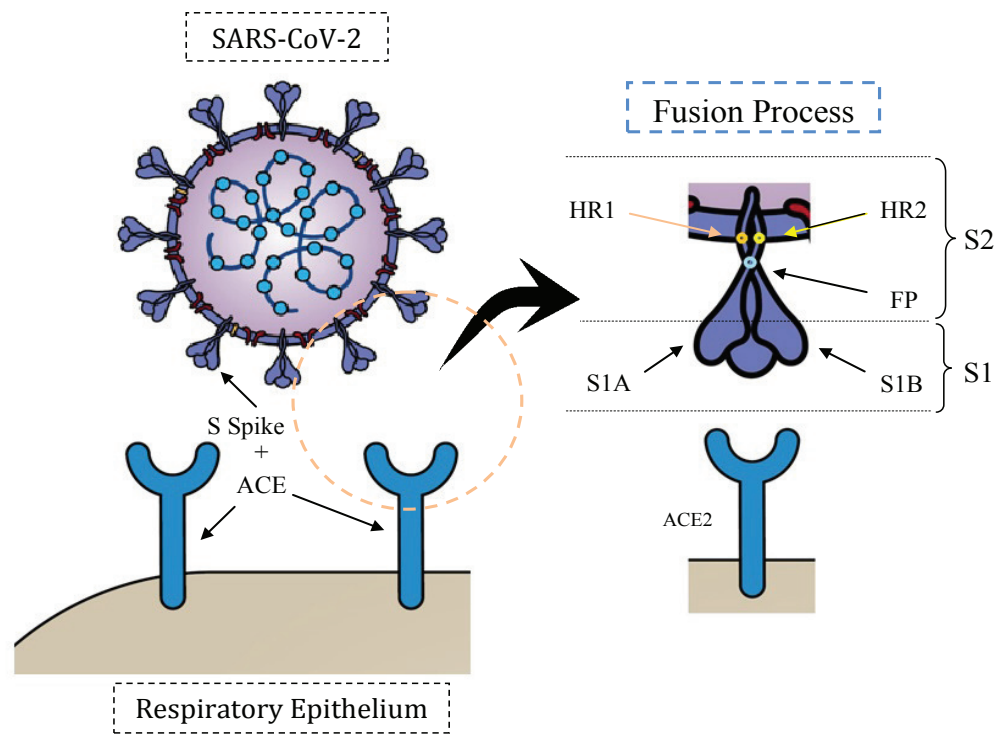

Figure 1: Left Panel: SARS-CoV-2 Cell Entry process in epithelial cells. Right Panel: Schematic diagram of SARS-CoV-2 spike. The spike protein consists of the $\mathrm{S} 1$ and $\mathrm{S} 2$ domains. The $\mathrm{S} 1$ domain contains the receptor bonding domain and responsible for primary bonding with host ACE2 receptor. The S2 domain, responsible for fusion process. The S2 receptor contains the fusion peptide (blue), the heptad repeat HR1 (orange) and HR2 (yellow).)

et al. (2012)). S protein is not only capable of neutralizing antibody but it also contains several immunogenic $\mathrm{T}$ cell epitopes. Some of the epitopes found in either S1 or S2 domain. These proteins are useful for SARS-CoV-2 vaccine development (see Li G. \& De Clercq, E., (2020)).

Spike is the main structural protein of the corona virus and assembles into a special corolla structure on the surface of the virus as a trimer. This protein interacts with the host by bonding to host cell receptors to mediate virus invasion and determine viral tissue or host tropism. SARS-CoV-2 spike (S) interact with host angiotensin-converting enzyme 2 (ACE2) receptors (see Fig.1). S1B receptor-bonding motifs (RBMs) (in green) bind ACE2 receptor. After that, the S1A domain may confer additional host interactions with the ACE2 receptor. A furin protease cleavage substrate that may confer heightened sensitivity to host protease cleavages (see Belouzard et al. (2009)). Subsequently, surface proteases cleave S2, the fusion-mediating 
subunit of $\mathrm{S}$, which triggers a series of conformational changes that result in the fusion between the viral envelope and the target cell membrane. During these conformational changes, the S2 subunit mediates virus-cell and cell-cell membrane fusion is to be occurred. At the same time, spike structural integrity and cleavage activation play a key role in virus invasion and virulence. Therapeutic strategies to block coronavirus from entering host cells by targeting spike proteins or specific receptors on the host surface are valuable for the development of anti-viral drugs.

Post-infection chloroquine (CQ) treatment is effective in preventing the spread of SARS-CoV infection into the body. When chloroquine was added after the initiation of infection, there was a dramatic dose-dependent decrease in the number of virus antigen-positive cells. Martin et al. (2020) identified chloroquine as an effective antiviral agent for SARS-CoV during cell culture conditions. Adding the drug prior to infection or after the initiation and establishment, the impairment of terminal glycosylation of ACE2 may result in reduced bonding affinities between ACE2 and SARS CoV spike protein and negatively influence the initiation of SARS-CoV infection (see Martin et al. (2020)). The experiment confirmed that Hydroxychloroquine (HCQ) effectively inhibited the entry step, as well as the post-entry stages of SARS-CoV-2, which was also found upon CQ treatment (see Savarino et al. (2006)). With a safe dosage, HCQ concentration in the liver, spleen, kidney, and lung tissues is likely to be achieved to inhibit SARS-CoV-2 infection (see Jia et al. (2020)).

$\mathrm{S} 2$ subunit plays a very important role in the membrane fusion process mediated by MERS-CoV or SARS-CoV S protein. Similarly, a recent study suggested that HR1 and HR2 in subunit S2 of SARS-CoV-2 also interacted to form a coiled-coil complex to support membrane fusion and viral infection.

The lipopeptide EK1C4 plays an inhibitory role against membrane fusion mediated by $\mathrm{S}$ proteins and entry of SARS-CoV-2 in epithelial cells. S protein-mediated cell-cell fusion could be effectively inhibited by EK1C4 with 266 IC50 of $4.3 \mathrm{nM}$ (see Jia et al. (2020)). EK1C4 has more advantages for the treatment and prevention of SARS-CoV-2 infection. It mainly targets the HR1 domain in the $\mathrm{S} 2$ subunit of $\mathrm{S}$ protein. EK1C4 possesses a high genetic barrier to resistance. EK1C4 can be used in an intranasal formulation to prevent COVID-19 infection. It can be used in inhalation formulation to reduce the viral loads in the lungs of the COVID-19 patients. Furthermore as EK1C4 is a peptide drug thus it is expected to be safe for humans. Because it will be used locally, not systemically. Thus EK1C4 shows exceptional promise to be developed as the first fusion inhibitor-based antiviral drug to prevent the pandemic COVID-19. 


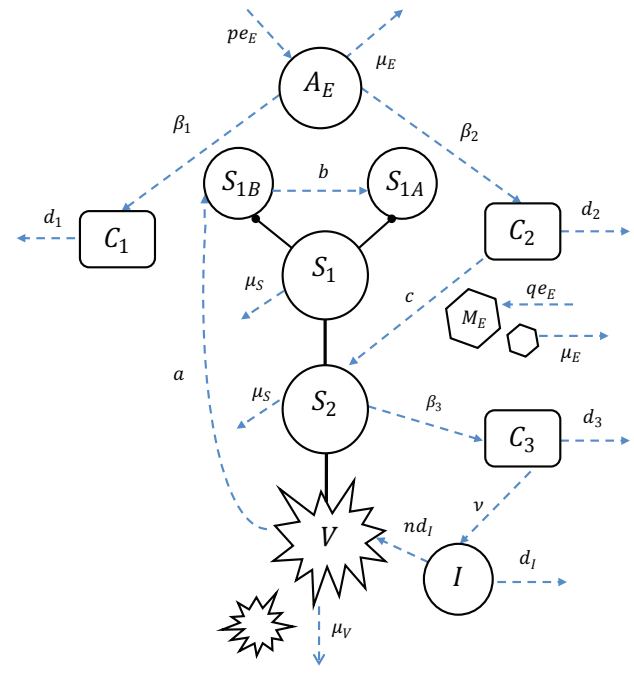

Figure 2: Schematic representation of the mathematical model (1) - (3).

Our mathematical model considers the interaction of the SARS-CoV2 virus with epithelial cells in order to study the dynamics of the fusion process and its inhibition using two different drugs: (1) HCQ (to block the SARS-CoV-2 S spike bonding with ACE2), and (2) fusion inhibitor lipopeptide EK1C4 (to prevent the SARS-CoV-2 viral entry into epithelial cells). We make use of impulsive differential equations to model the change in drug concentration which occurs when a new dose is administered. These technique allows us to make a number of interesting predictions. This paper is organised as follows. In Section 2, we formulate our mathematical model following the infection process. In Section 3, we examine the model without drugs and give some numerical evidence in support of our results. In Section 4, we state some analytical results for the model with drugs. Few numerical simulations are also done here. Finally, in Section 5 we discuss the biological implications of the model predictions.

\section{Model formulation}

To form the mathematical model, we follow a step-by-step infection process, described as earlier. For this, we consider $V$ as the concentration of SARSCoV-2 and $A_{E}$ as the concentration of ACE2 receptor on the epithelial cell surface, produced at the rate $p e_{E}$, where $e_{E}$ is the source of susceptible epithelial cells and $p$ is the number of ACE2 receptors. When the virus 
enters into the host body, the viral subunits of $\mathrm{S}$ protein $\mathrm{S} 1 \mathrm{~A}$ and $\mathrm{S} 1 \mathrm{~B}$, denoted by $S_{1 A}, S_{1 B}$ respectively, get activated and attached to $A_{E}$ cells to form the dimeric complexes $C_{1}$ of $\mathrm{S} 1 \mathrm{~B}$ and $\mathrm{ACE} 2$ receptor, and $C_{2}$ of $\mathrm{S} 1 \mathrm{~A}$ and ACE2 receptor at the rates $\beta_{1}$ and $\beta_{2}$, respectively. $b$ represents the successful exposure of $S_{1 B}$, since it is exposed only after attachment between S1B and ACE2 receptor is complete. The dynamics of primary infection stage of SARS-CoV-2 thus given by:

$$
\begin{aligned}
\frac{d S_{1 B}}{d t} & =a V-\mu_{S} S_{1 B}-\beta_{1} S_{1 B} A_{E}, \\
\frac{d A_{E}}{d t} & =p e_{E}-\mu_{E} A_{E}-\beta_{1} S_{1 B} A_{E}-\beta_{2} S_{1 A} A_{E} \\
\frac{d C_{1}}{d t} & =\beta_{1} S_{1 B} A_{E}-d_{1} C_{1}, \\
\frac{d S_{1 A}}{d t} & =b C_{1}-\mu_{S} S_{1 A}-\beta_{2} S_{1 A} A_{E}, \\
\frac{d C_{2}}{d t} & =\beta_{2} S_{1 A} A_{E}-d_{2} C_{2} .
\end{aligned}
$$

Here, $a$ represents the multiplication capacity of $S_{1 B}$ in response to virus. The parameter $\mu_{S}$ denotes the decay of $S_{1 B}$ and $S_{1 A}$ subunits of S, and $d_{1}$, $d_{2}$ are the dissociation rates of $C_{1}, C_{2}$ respectively.

This additional attachment between $\mathrm{S} 1 \mathrm{~A}$ and ACE2 results the successful exposure of viral fusion protein, $S_{2}$ at a rate $c$, which allows the surface protease on epithelial cells membrane, $M_{E}$ to bind with it at the bonding force $\beta_{3}$ to produce the of $S_{2}$ and $S_{1}-A_{E}$ ternary complex, $C_{3}$.

$$
\begin{aligned}
\frac{d S_{2}}{d t} & =c C_{2}-\mu_{S} S_{2}-\beta_{3} S_{2} M_{E} \\
\frac{d M_{E}}{d t} & =q e_{E}-\mu_{E} M_{E}-\beta_{3} S_{2} M_{E} \\
\frac{d C_{3}}{d t} & =\beta_{3} S_{2} M_{E}-d_{3} C_{3}
\end{aligned}
$$

where, $q$ denotes the number of surface protease on one epithelial cell. $\mu_{S}$ and $\mu_{E}$ denote the decay of $S_{2}$ subunits and healthy epithelial cells. The dissociation rate of $C_{3}$ complex is $d_{3}$.

The formation of $C_{3}$ complex ultimately turns out the infection of epithelial cells, $I$ and production of new viruses at the rates $\nu$ and $n d_{I}$, respectively. $n$ represents the number of virus particles that are produced by one 
infected epithelial cell and $d_{I}$ is the death rate of infected epithelial cells. The clearance rate of free SARS-CoV-2 is denoted by $\mu_{V}$.

$$
\begin{aligned}
\frac{d I}{d t} & =\nu C_{3}-d_{I} I, \\
\frac{d V}{d t} & =n d_{I} I-\mu_{V} V .
\end{aligned}
$$

We ignore the loss of virions due to reinfection of infected cells. This has the effect of overestimating the virion count and plays no important role in the estimates below. The schematic diagram is shown in FIG.2.

\section{Properties of the system}

The system (1) - (3) has two equilibrium points, namely

- the disease free equilibrium $\hat{E}\left(\hat{S}_{1 B}, \hat{A}_{E}, 0, \hat{C}_{1}, \hat{S}_{1 A}, \hat{C}_{2}, \hat{S}_{2}, \hat{M}_{E}, \hat{C}_{3}, \hat{I}, \hat{V}\right)=$ $\left(0, \frac{p e_{E}}{\mu_{E}}, 0,0,0,0,0, \frac{q e_{E}}{\mu_{E}}, 0,0,0\right)$,

- the endemic equilibrium $E^{*}\left(S_{1 B}^{*}, A_{E}^{*}, 0, C_{1}^{*}, S_{1 A}^{*}, C_{2}^{*}, S_{2}^{*}, M_{E}^{*}, C_{3}^{*}, I^{*}, V^{*}\right)$.

\subsection{Analysis of the disease free state}

The linearization of the first, third, forth, fifth, sixth, eighth, ninth, and tenth equations of the model (1) - (3) at the disease free state $\hat{E}$ can be rewritten in the following form:

$$
\frac{d W}{d t}=\left(X_{\hat{E}}-Y_{\hat{E}}\right) W
$$

where

$$
\begin{gathered}
W=\left[S_{1 B}, C_{1}, S_{1 A}, C_{2}, S_{2}, C_{3}, I, V\right]^{T}, \\
X_{\hat{E}}=\left(\begin{array}{cccccccc}
0 & 0 & 0 & 0 & 0 & 0 & 0 & a \\
\beta_{1} \hat{A}_{E} & 0 & 0 & 0 & 0 & 0 & 0 & 0 \\
0 & b & 0 & 0 & 0 & 0 & 0 & 0 \\
0 & 0 & \beta_{2} \hat{A}_{E} & 0 & 0 & 0 & 0 & 0 \\
0 & 0 & 0 & c & 0 & 0 & 0 & 0 \\
0 & 0 & 0 & 0 & \beta_{3} \hat{M}_{E} & 0 & 0 & 0 \\
0 & 0 & 0 & 0 & 0 & \nu & 0 & 0 \\
0 & 0 & 0 & 0 & 0 & 0 & n d_{I} & 0
\end{array}\right),
\end{gathered}
$$


Table 1: Set of parameters values used for numerical simulations

\begin{tabular}{|c|c|c|c|}
\hline Parameter & $\overline{\text { Explanation }}$ & $\begin{array}{c}\text { Assigned } \\
\text { value }\end{array}$ & $\overline{\text { Unit }}$ \\
\hline $\bar{a} a$ & Multiplication capacity of S1B & 50 & $d a y^{-1}$ \\
\hline$b$ & Exposure rate of S1A & 42 & $d a y^{-1}$ \\
\hline$c$ & Exposure rate of $\mathrm{S} 2$ & 25 & $d a y^{-1}$ \\
\hline$\beta_{1}$ & Bonding force between & $5 \times 10^{-5}$ & $m m^{-3} d a y^{-1}$ \\
\hline$\beta_{2}$ & $\begin{array}{l}\text { S1B and ACE2 receptor } \\
\text { Bonding force between } \\
\text { S1A and ACE2 receptor }\end{array}$ & $5 \times 10^{-5}$ & $m m^{-3} d a y^{-1}$ \\
\hline$\beta_{3}$ & $\begin{array}{l}\text { Bonding force between } \\
\text { S2 and surface protein }\end{array}$ & $5 \times 10^{-5}$ & $m m^{-3} d a y^{-1}$ \\
\hline$\mu_{S}$ & $\begin{array}{l}\text { Decay rate of } \mathrm{S} 1 \mathrm{~A}, \mathrm{~S} 1 \mathrm{~B}, \\
\mathrm{~S} 2 \text { subunit of } \mathrm{S} \text { protein }\end{array}$ & 5 & $d a y^{-1}$ \\
\hline$\mu_{E}$ & Death rate of epithelial cell & 0.05 & $d a y^{-1}$ \\
\hline$\mu_{V}$ & Clearance rate of virus & 2 & $d a y^{-1}$ \\
\hline$d_{1}$ & Dissociation rate of $C_{1}$ & 0.4 & $d a y^{-1}$ \\
\hline$d_{2}$ & Dissociation rate of $C_{2}$ & 0.3 & $d a y^{-1}$ \\
\hline$d_{3}$ & Dissociation rate of $C_{3}$ & 0.3 & $d a y^{-1}$ \\
\hline$p$ & $\begin{array}{l}\text { Number of ACE2 receptors } \\
\text { on one epithelial cell }\end{array}$ & 1 & cell $^{-1}$ \\
\hline$q$ & Number of surface receptor & 0.5 & cell $^{-1}$ \\
\hline$e_{E}$ & $\begin{array}{l}\text { Source rate of susceptible } \\
\text { epithelial cell }\end{array}$ & 80 & $d a y^{-1} m m^{-3}$ \\
\hline$n$ & $\begin{array}{l}\text { Rate of production of } \\
\text { virions per infected cell }\end{array}$ & 540 & \\
\hline$\nu$ & Infection rate of infected cell & 0.6 & $d a y^{-1}$ \\
\hline$m_{1}$ & Blocking rate of ACE2 receptor & 0.1 & $d a y^{-1}$ \\
\hline$m_{2}$ & $\begin{array}{c}\text { Rate at which HCQ are cleared from } \\
\text { intracellular compartments }\end{array}$ & 0.1 & $\mu g m m^{-3}$ \\
\hline$I C_{50}$ & $\begin{array}{l}\text { Concentration of fusion } \\
\text { viral replication by } 50 \%\end{array}$ & $10^{-5}$ & $\mu \mathrm{gmm}^{-3}$ \\
\hline$\omega_{1}$ & Rate at which HCQ is cleared & 0.5 & $d a y^{-1}$ \\
\hline$\omega_{2}$ & Rate at which fusion inhibitor is cleared & 0.5 & $d a y^{-1}$ \\
\hline
\end{tabular}


and

$$
Y_{\hat{E}}=\left(\begin{array}{cccccccc}
\left(\mu_{S}+\beta_{1} \hat{A}_{E}\right) & 0 & 0 & 0 & 0 & 0 & 0 & 0 \\
0 & d_{1} & 0 & 0 & 0 & 0 & 0 & 0 \\
0 & 0 & \left(\mu_{S}+\beta_{2} \hat{A}_{E}\right) & 0 & 0 & 0 & 0 & 0 \\
0 & 0 & 0 & d_{2} & 0 & 0 & 0 & 0 \\
0 & 0 & 0 & 0 & \left(\mu_{S}+\beta_{3} \hat{M}_{E}\right) & 0 & 0 & 0 \\
0 & 0 & 0 & 0 & 0 & d_{3} & 0 & 0 \\
0 & 0 & 0 & 0 & 0 & 0 & d_{I} & 0 \\
0 & 0 & 0 & 0 & 0 & 0 & 0 & \mu_{V}
\end{array}\right) .
$$

A threshold criteria, $R_{0}$, can be derived using the spectral radius of next generation matrix. Here $R_{0}$ is called the basic reproduction number. To find $R_{0}$, we must find the largest eigenvalue of $X_{\hat{E}} Y_{\hat{E}}^{-1}$.

Thus,

$$
R_{0}=\rho\left(X_{\hat{E}} Y_{\hat{E}}^{-1}\right)
$$

$=\max _{|\lambda|} \operatorname{det}\left(\begin{array}{cccccccc}\lambda & 0 & 0 & 0 & 0 & 0 & 0 & -\frac{a}{\mu_{V}} \\ -\frac{\beta_{1} \hat{A}_{E}}{\mu_{S}+\beta_{1} \hat{A}_{E}} & \lambda & 0 & 0 & 0 & 0 & 0 & 0 \\ 0 & -\frac{b}{d_{1}} & \lambda & 0 & 0 & 0 & 0 & 0 \\ 0 & 0 & -\frac{\beta_{2} \hat{A}_{E}}{\mu_{S}+\beta_{2} \hat{A}_{E}} & \lambda & 0 & 0 & 0 & 0 \\ 0 & 0 & 0 & -\frac{c}{d_{2}} & \lambda & 0 & 0 & 0 \\ 0 & 0 & 0 & 0 & -\frac{\beta_{3} \hat{M}_{E}}{\mu_{S}+\beta_{3} \hat{M}_{E}} & \lambda & 0 & 0 \\ 0 & 0 & 0 & 0 & 0 & -\frac{\nu}{d_{3}} & \lambda & 0 \\ 0 & 0 & 0 & 0 & 0 & 0 & -n & \lambda\end{array}\right)$.

The characteristic equation of $X_{\hat{E}} Y_{\hat{E}}^{-1}$ is

$$
\lambda^{8}-\frac{n \nu a b c \beta_{1} \beta_{2} \beta_{3} \hat{A}_{E}^{2} \hat{M}_{E}}{d_{1} d_{2} d_{3} \mu_{V}\left(\mu_{S}+\beta_{1} \hat{A}_{E}\right)\left(\mu_{S}+\beta_{2} \hat{A}_{E}\right)\left(\mu_{S}+\beta_{3} \hat{M}_{E}\right)}=0 .
$$

Then

$$
\begin{aligned}
R_{0} & =\left[\frac{n \nu a b c \beta_{1} \beta_{2} \beta_{3} \hat{A}_{E}^{2} \hat{M}_{E}}{d_{1} d_{2} d_{3} \mu_{V}\left(\mu_{S}+\beta_{1} \hat{A}_{E}\right)\left(\mu_{S}+\beta_{2} \hat{A}_{E}\right)\left(\mu_{S}+\beta_{3} \hat{M}_{E}\right)}\right]^{\frac{1}{8}} \\
& =\left[\frac{n \nu a b c \beta_{1} \beta_{2} \beta_{3} p^{2} q e_{E}^{3}}{d_{1} d_{2} d_{3} \mu_{V}\left(\mu_{S} \mu_{E}+\beta_{1} p e_{E}\right)\left(\mu_{S} \mu_{E}+\beta_{2} p e_{E}\right)\left(\mu_{S} \mu_{E}+\beta_{3} q e_{E}\right)}\right]^{\frac{1}{8}}{ }_{(6)}
\end{aligned}
$$


Remark: The disease free equilibrium $\hat{E}$ always exists and it is locally stable if $R_{0}<1$ and unstable, if $R_{0}>1$. To verify the persistence of infection, we use the threshold value of $R_{0}$.

\subsubsection{The Global stability}

Theorem 1. When $R_{0} \leq 1$, the disease free equilibrium $\hat{E}$ is globally asymptotically stable and it is the unique equilibrium. Otherwise, $\hat{E}$ is unstable and there exists a unique endemic equilibrium.

Proof. In absence of SARS-CoV-2, the concentration of $A_{E}$ and $M_{E}$ is defined as

$$
\begin{aligned}
\frac{d A_{E}}{d t} & =p e_{E}-\mu_{E} A_{E} \\
\Rightarrow A_{E}(t) & =\frac{p e_{E}}{\mu_{E}}-\left(\frac{p e_{E}}{\mu_{E}}-A_{E}(0)\right) e^{-\mu_{E} t} .
\end{aligned}
$$

When $t \rightarrow \infty, \quad A_{E}(t) \rightarrow \frac{p e_{E}}{\mu_{E}}$.

Hence the initial value $A_{E}(0)<\frac{p e_{E}}{\mu_{E}}$. Then all trajectories must lies below $\frac{p e_{E}}{\mu_{E}}$. Conversely, if the initial value satisfy $A_{E}(0)>\frac{p e_{E}}{\mu_{E}}$, then all the trajectories must remain above $\frac{p e_{E}}{\mu_{E}}$. Suppose the system are at or below its steady state, then the system must satisfy the inequality $A_{E}(t) \leq \frac{p e_{E}}{\mu_{E}}$. In similar way we can find, $M_{E}(t) \leq \frac{q e_{E}}{\mu_{E}}$.

To prove the global stability, we take a Lyapunov function define as

$$
\mathcal{L}=\mathcal{A} S_{1 B}+\mathcal{B} C_{1}+\mathcal{C} S_{1 A}+\mathcal{D} C_{2}+\mathcal{E} S_{2}+\mathcal{F} C_{3}+\mathcal{G} I+\mathcal{H} V
$$

where $\mathcal{A}, \mathcal{B}, \mathcal{C}, \mathcal{D}, \mathcal{E}, \mathcal{F}, \mathcal{G}, \mathcal{H}$ all are positive.

Then

$$
\begin{aligned}
\frac{d \mathcal{L}}{d t}= & \left(\mathcal{A} a-\mathcal{H} \mu_{V}\right) V+\left(\mathcal{C} b-\mathcal{B} d_{1}\right) C_{1}+\left(\mathcal{E} c-\mathcal{D} d_{2}\right) C_{2}+\left(\mathcal{G} \nu-\mathcal{F} d_{3}\right) C_{3}+\left(\mathcal{H} n d_{I}-\mathcal{G} d_{I}\right) I \\
& +(\mathcal{B}-\mathcal{A}) \beta_{1} S_{1 B} A_{E}+(\mathcal{D}-\mathcal{C}) \beta_{2} S_{1 A} A_{E}+(\mathcal{F}-\mathcal{E}) \beta_{3} S_{2} M_{E}-\mu_{S}\left(\mathcal{A} S_{1 B}+\mathcal{C} S_{1 A}+\mathcal{E} S_{2}\right) \\
\leq & \left(\mathcal{A} a-\mathcal{H} \mu_{V}\right) V+\left(\mathcal{C} b-\mathcal{B} d_{1}\right) C_{1}+\left(\mathcal{E} c-\mathcal{D} d_{2}\right) C_{2}+\left(\mathcal{G} \nu-\mathcal{F} d_{3}\right) C_{3}+\left(\mathcal{H} n d_{I}-\mathcal{G} d_{I}\right) I \\
& +\left[(\mathcal{B}-\mathcal{A}) \frac{\beta_{1} p e_{E}}{\mu_{E}}-\mathcal{A} \mu_{S}\right] S_{1 B}+\left[(\mathcal{D}-\mathcal{C}) \frac{\beta_{2} p e_{E}}{\mu_{E}}-\mathcal{C} \mu_{S}\right] S_{1 A}+\left[(\mathcal{F}-\mathcal{E}) \frac{\beta_{3} q e_{E}}{\mu_{E}}-\mathcal{E} \mu_{S}\right] S_{2} .
\end{aligned}
$$

We can choose proper positive numbers $\mathcal{A}, \mathcal{B}, \mathcal{C}, \mathcal{D}, \mathcal{E}, \mathcal{F}, \mathcal{G}, \mathcal{H}$ as follows: 


$$
\begin{aligned}
\mathcal{A} & =\frac{\mu_{V}}{n a}, \quad \mathcal{B}=\frac{\mu_{V}\left(\mu_{S} \mu_{E}+\beta_{1} p e_{E}\right)}{n a p e_{E} \beta_{1}}, \quad \mathcal{C}=\frac{d_{1} \mu_{V}\left(\mu_{S} \mu_{E}+\beta_{1} p e_{E}\right)}{n a b p e_{E} \beta_{1}}, \\
\mathcal{D} & =\frac{d_{1} \mu_{V}\left(\mu_{S} \mu_{E}+\beta_{1} p e_{E}\right)\left(\mu_{S} \mu_{E}+\beta_{2} p e_{E}\right)}{n a b p^{2} e_{E}^{2} \beta_{1} \beta_{2}}, \quad \mathcal{G}=1, \quad \mathcal{H}=\frac{1}{n}, \\
\mathcal{E} & =\frac{d_{1} d_{2} \mu_{V}\left(\mu_{S} \mu_{E}+\beta_{1} p e_{E}\right)\left(\mu_{S} \mu_{E}+\beta_{2} p e_{E}\right)}{n a b c p^{2} e_{E}^{3} \beta_{1} \beta_{2}}, \\
\mathcal{F} & =\frac{d_{1} d_{2} \mu_{V}\left(\mu_{S} \mu_{E}+\beta_{1} p e_{E}\right)\left(\mu_{S} \mu_{E}+\beta_{2} p e_{E}\right)\left(\mu_{S} \mu_{E}+\beta_{3} q e_{E}\right)}{n a b c p^{2} q e_{E}^{3} \beta_{1} \beta_{2} \beta_{3}} .
\end{aligned}
$$

Hence ,

$$
\begin{aligned}
\frac{d \mathcal{L}}{d t} & \leq\left(\nu \mathcal{G}-d_{3} \mathcal{F}\right) C_{3} \\
& =\left[\nu-\frac{d_{1} d_{2} d_{3} \mu_{V}\left(\mu_{S} \mu_{E}+\beta_{1} p e_{E}\right)\left(\mu_{S} \mu_{E}+\beta_{2} p e_{E}\right)\left(\mu_{S} \mu_{E}+\beta_{3} q e_{E}\right)}{\operatorname{nabcp}^{2} q e_{E}^{3} \beta_{1} \beta_{2} \beta_{3}}\right] C_{3} \\
& =\frac{\nu}{R_{0}^{8}}\left[R_{0}^{8}-1\right] C_{3}
\end{aligned}
$$

When $R_{0} \leq 1$ then $\frac{d \mathcal{L}}{d t} \leq 0$. Also if $C_{3}=0, \frac{d \mathcal{L}}{d t}=0$, then from (12) get that $I \rightarrow 0, \quad V \rightarrow 0, \quad S_{1 B} \rightarrow 0, \quad S_{1 A} \rightarrow 0, \quad S_{2} \rightarrow 0, \quad C_{1} \rightarrow 0, \quad C_{2} \rightarrow 0$, respectively when $t \rightarrow \infty$. Hence, accordingly to Lyapunov-Lasalle theorem (see Hethcote, H. W.,(2000)), $R_{0} \leq 1$ which implies the disease free equilibrium is globally asymptotically stable. This completes the proof. 


\subsection{The endemic state}

The endemic periodic orbit is in the form

$E^{*}\left(S_{1 B}^{*}, A_{E}^{*}, 0, C_{1}^{*}, S_{1 A}^{*}, C_{2}^{*}, S_{2}^{*}, M_{E}^{*}, C_{3}^{*}, I^{*}, V^{*}\right)$, where

$$
\begin{aligned}
V^{*} & =\frac{n d_{I} I^{*}}{\mu_{V}}, \\
C_{3}^{*} & =\frac{d_{I} I^{*}}{\nu} \\
M_{E}^{*} & =\frac{q e_{E} \nu-d_{3} d_{I} I^{*}}{\mu_{E} \nu}, \\
S_{2}^{*} & =\frac{\mu_{E} d_{3} d_{I} I^{*}}{\beta_{3}\left(q e_{E} \nu-d_{3} d_{I} I^{*}\right)} \\
C_{2}^{*} & =\frac{d_{3} d_{I}}{c}\left[\frac{\mu_{S} \mu_{E}}{\beta_{3}\left(q e_{E} \nu-d_{3} d_{I} I^{*}\right)}+\frac{1}{\nu}\right] I^{*}, \\
S_{1 B}^{*} & =\frac{a n d_{I} I^{*}}{\mu_{V}\left(\mu_{S}+\beta_{1} A_{E}^{*}\right)}, \\
S_{1 A}^{*} & =\frac{a b n d_{I} \beta_{1} A_{E}^{*} I^{*}}{d_{2}\left(\mu_{S}+\beta_{1} A_{E}^{*}\right)\left(\mu_{S}+\beta_{2} A_{E}^{*}\right)}, \\
I^{*} & =\frac{\nu\left(P_{1}+P_{2} A_{E}^{*}+P_{3}\left(A_{E}^{*}\right)^{2}\right)}{d_{3} d_{I} \beta_{3}\left(P_{4}+P_{5} A_{E}^{*}+P_{6}\left(A_{E}^{*}\right)^{2}\right)},
\end{aligned}
$$

where, $P_{1}=d_{2}^{2} d_{3} \mu_{S}^{2}\left(q e_{E} \beta_{3}+\mu_{S} \mu_{E}\right), \quad P_{2}=d_{2}^{2} d_{3} \mu_{S}\left(\beta_{1}+\beta_{2}\right)\left(q e_{E} \beta_{3}+\right.$ $\left.\mu_{S} \mu_{E}\right), P_{3}=d_{2}^{2} d_{3} \beta_{1} \beta_{2}\left(q e_{E} \beta_{3}+\mu_{S} \mu_{E}\right)-a b c n \nu q e_{E} \beta_{1} \beta_{2} \beta_{3} P_{4}=d_{2}^{2} d_{3} \mu_{S}^{2}, \quad P_{5}=$ $d_{2}^{2} d_{3} \mu_{S}\left(\beta_{1}+\beta_{2}\right), \quad P_{6}=\beta_{1} \beta_{2}\left(d_{2}^{2} d_{3}-a b c n \nu\right)$.

The value of $A_{E}^{*}$ (if it exists) can be found from the equation,

$$
\begin{aligned}
f\left(A_{E}^{*}\right) & =Q_{1}\left(A_{E}^{*}\right)^{5}+Q_{2}\left(A_{E}^{*}\right)^{4}+Q_{3}\left(A_{E}^{*}\right)^{3}+Q_{4}\left(A_{E}^{*}\right)^{2}+Q_{5} A_{E}^{*}+Q_{6} \\
& =0
\end{aligned}
$$




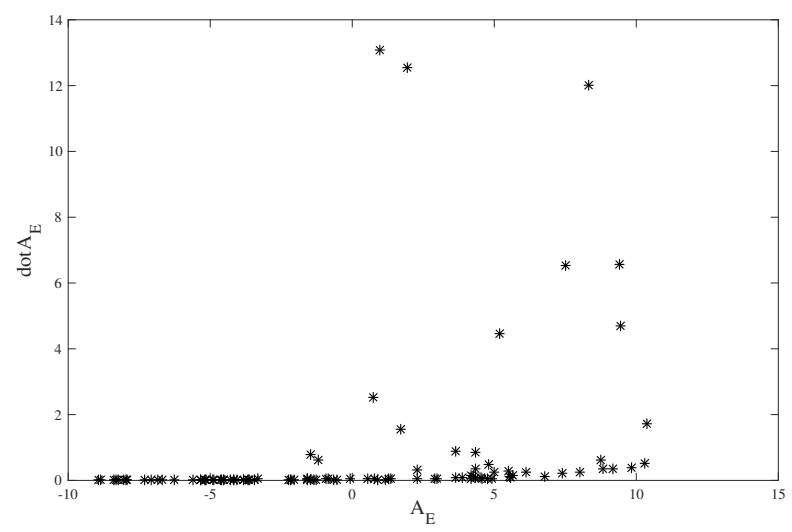

Figure 3: Poincaré section for the set of parameter values given in the Table. 1

where,

$$
\begin{aligned}
Q_{1}= & p e_{E} d_{2} d_{3} \mu_{S}^{4}>0 \\
Q_{2}= & \mu_{E}\left\{\mu_{S}\left(\beta_{1}+\beta_{2}\right) P_{6}+\beta_{1} \beta_{2} P_{5}\right\}-p e_{E} \beta_{1} \beta_{2} P_{6} \\
Q_{3}= & \mu_{E}\left\{\mu_{S}^{2} P_{6}+\beta_{1} \beta_{2} P_{4}+\mu_{S}\left(\beta_{1}+\beta_{2}\right) P_{5}\right\} \\
& +\frac{a n \nu \beta_{1}}{d_{2} d_{3} \mu_{V} \beta_{3}}\left\{d_{2} \mu_{S} P_{3}+\beta_{2}\left(d_{2}+b \mu_{V}\right) P_{2}\right\}-p e_{E}\left\{\mu_{S}\left(\beta_{1}+\beta_{2}\right) P_{6}+\beta_{1} \beta_{2} P_{5}\right\}, \\
Q_{4}= & \mu_{S} \mu_{E}\left\{\mu_{S} P_{5}+\left(\beta_{1}+\beta_{2}\right) P_{4}\right\}+\frac{a n \nu \beta_{1}}{d_{2} d_{3} \beta_{3} \mu_{V}}\left\{d_{2} \mu_{S} P_{2}+\left(d_{2}+b \mu_{V}\right) \beta_{2} P_{1}\right\} \\
& -p e_{E}\left\{\mu_{S}^{2} P_{6}+\beta_{1} \beta_{2} P_{4}+\mu_{S}\left(\beta_{1}+\beta_{2}\right) P_{5}\right\}, \\
Q_{5}= & \mu_{E} \mu_{S}^{2} P_{4}+\frac{a n \nu \mu_{S} \beta_{1} P_{1}}{d_{3} \mu_{V} \beta_{3}}-p e_{E} \mu_{S}\left\{\left(\beta_{1}+\beta_{2}\right) P_{4}+\mu_{S} P_{5}\right\}, \\
Q_{6}= & -\beta_{1} \beta_{2} \mu_{E} P_{6} .
\end{aligned}
$$

Now, to show the existence of a positive endemic equilibrium $E^{*}$ of system (1) - (3), we use Descartes' rule of signs. Following this rule, we concluded that equation (10) will have a unique positive root either if (i) $Q_{i}<0$, for $i=2,3,4,5,6$ or (ii) $Q_{i}>0$ for $i=2,3,4,5$ and $Q_{6}<0$.

Remark: If $R_{0}>1$, the disease free orbit is unstable. Although the endemic periodic orbit $E^{*}$ exists when $R_{0}>1$, it may or may not be stable, depending on the parameters. If it is unstable, then we may have higher-order behavior, such as higher-order periodicity or chaos. If $R_{0}<1$ fluctuates around 1 , the results are indeterminate. To check this, we have 


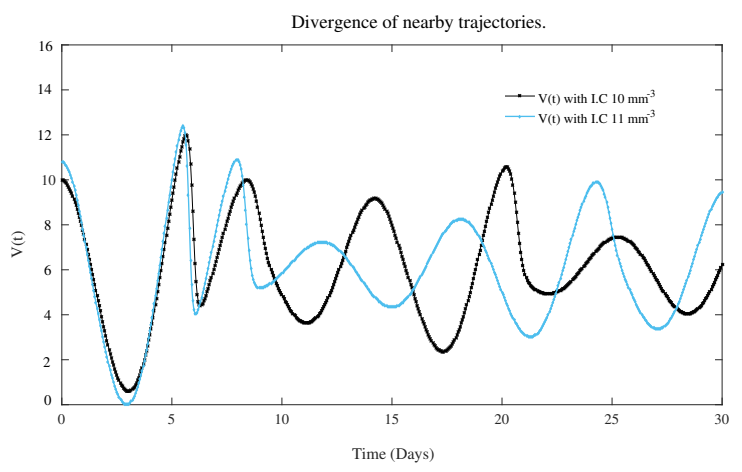

Figure 4: Two trajectories for the system (1) - (3) with initial conditions $\mathrm{V}(0)=10$ and $\mathrm{V}(0)=12$ diverge appreciably after $t=6^{\text {th }}$ day.

plotted Poincaré section for the equation (10) in Fig.3 for the specified parameter values given in the Table.1. This picture shows the system is chaotic and the Poincaré section is a fractal set. It is clear that while the points are not repeating, they are not completely randomly distributed in space. In the next figure (Fig.4), the divergence of two trajectories is shown and it states that initially they start quite close to each other with initial conditions $V(0)=10$ and $V(0)=12$, but after 6 th day of infection, it starts to diverge appreciably, which is quite relevant with the real scenario. Lyapunov exponents are a measure of the exponential rate of divergence of two trajectories that begin arbitrarily close to each other. A Lyapunov exponent greater than zero signifies the system to be chaotic. In Fig.5, we plot all the Lyapunov exponents for the system (1) - (3). The largest is greater than zero and, therefore, the system is chaotic.

\section{The system in the presence of drugs}

We use $\mathrm{R}$ and $\mathrm{F}$ to denote the intracellular HCQ and fusion inhibitor lipopeptide EK1C4 drug concentrations in plasma. We assume that the effect of the medication is instantaneous, resulting in a system of impulsive differential equations (see Lou \& Smith (2011), Chatterjee \& Roy (2012), Roy et al. (2016)), whereby solutions are continuous for $t \neq t_{k}$ and $t \neq s_{k}$ and undergo an instantaneous change in state when $t=t_{k}$ and $t=s_{k}, k=$ $1,2,3, \ldots$. This technique assumes that the change in drug concentration immediately after a drug is administered is nearly instantaneous; that is, the 


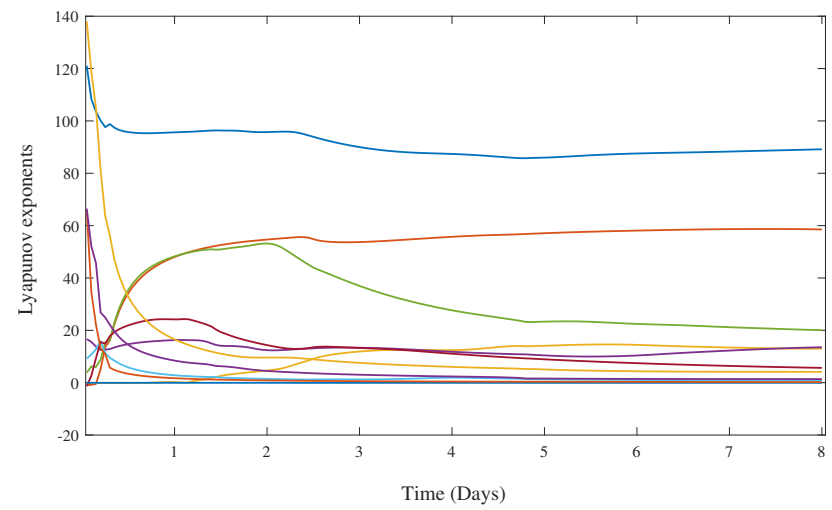

Figure 5: Plot of all the Lyapunov exponents for the system (1)-(3).

time-to-peak is negligible on the relevant time scale. By neglecting the dispersion and delay as the drug enters the body, we overestimate the temporal effects of medication at intervals.

The dynamics of the above system with drug is constructed following 
the schematic diagram (6) and is given by:

$$
\begin{aligned}
\frac{d S_{1 B}}{d t} & =a V-\mu_{S} S_{1 B}-\beta_{1} S_{1 B} A_{E} \\
\frac{d A_{E}}{d t} & =p e_{E}-\mu_{E} A_{E}-\beta_{1} S_{1 B} A_{E}-\beta_{2} S_{1 A} A_{E}-m_{1} A_{E} R+m_{2} A_{E}^{\prime} \\
\frac{d A_{E}^{\prime}}{d t} & =m_{1} A_{E} R-m_{2} A_{E}^{\prime}-\mu_{E} A_{E}^{\prime} \\
\frac{d C_{1}}{d t} & =\beta_{1} S_{1 B} A_{E}-d_{1} C_{1} \\
\frac{d S_{1 A}}{d t} & =b C_{1}-\mu_{S} S_{1 A}-\beta_{2} S_{1 A} A_{E} \\
\frac{d C_{2}}{d t} & =\beta_{2} S_{1 A} A_{E}-d_{2} C_{2} \\
\frac{d S_{2}}{d t} & =c C_{2}-\mu_{S} S_{2}-(1-\eta) \beta_{3} S_{2} M_{E} \\
\frac{d M_{E}}{d t} & =q e_{E}-\mu_{E} M_{E}-(1-\eta) \beta_{3} S_{2} M_{E} \\
\frac{d C_{3}}{d t} & =(1-\eta) \beta_{3} S_{2} M_{E}-d_{3} C_{3} \\
\frac{d I}{d t} & =\nu C_{3}-d_{I} I \\
\frac{d V}{d t} & =n d_{I} I-\mu_{V} V
\end{aligned}
$$

with

$$
\begin{array}{ll}
\frac{d R}{d t}=-\omega_{1} R, & t \neq t_{k}, \\
\frac{d F}{d t}=-\omega_{2} F, & t \neq s_{k},
\end{array}
$$

where, $\omega_{i},(i=1,2)$ represents the rate at which the drugs are cleared from intracellular compartments. In general $t_{k} \neq s_{k}$, so that the two drugs are taken at different times.

We consider that the concentration of ACE2 on epithelial cells is blocked by the HCQ (or R) drug and it is represented by $A_{E}^{\prime} \cdot m_{1}$ is the rate at which ACE2 receptors are blocked and drug effects wear off at rate $m_{2}$. The drug effectiveness of fusion inhibitor, $\eta$, is the degree to which the primary bonding forces $\beta_{3}$ is reduced by the current drug concentration, with $0<\eta<1$. We use Michaelis-Menten dynamics to model changes in $\eta$ with changing drug concentrations, $\eta=\frac{F(t)}{F(t)+I C_{50}}$ (see Lindi M. W. and 


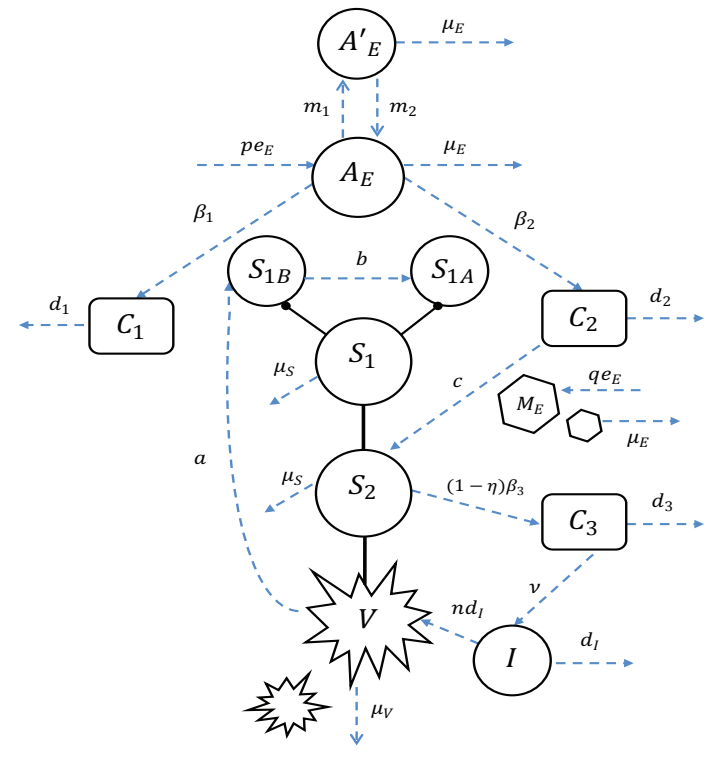

Figure 6: Schematic representation of the mathematical model (12) with (13-16).

Martin A. N., (2000), Lou \& Smith (2011)), where $F(t)$ is the intracellular drug concentration in plasma, and $I C_{50}$ is the concentration necessary to inhibit viral replication by $50 \%$. Here $(1-\eta(t))=\frac{I C_{50}}{I C_{50}+F(t)}$ represents the degree to which the fusion inhibitor block the $M_{E}$ and $S_{2}$ form fusion together. $\tilde{F}$ is the dosage (a constant) that is taken at each impulse time $t=s_{k},(k=1,2,3, \ldots)$. Since the drug may be taken at regular or irregular intervals, hence the drug time $t=s_{k}$ may not be fixed.

The impulsive conditions are

$$
\begin{array}{ll}
R\left(t_{k}^{+}\right)=\tilde{R}+R\left(t_{k}^{-}\right), & t=t_{k}, \\
F\left(s_{k}^{+}\right)=\tilde{F}+F\left(s_{k}^{-}\right), & t=s_{k} .
\end{array}
$$

Thus (12)-(16) describe a system of impulsive differential equations.

The drug dose, $\tilde{R}$, (or $\tilde{F}$ ) that is taken at each impulse time $t_{k}$ (or $s_{k}$ ) $(k=1,2,3 \ldots)$ is kept constant. Since drugs may be taken at either regular or irregular intervals, we have considered impulse time $t_{k}$ (or $s_{k}$ ) to be fixed.

The time between two successful doses are well defined as $\tau \equiv t_{k+1}-t_{k}$ 
and $\sigma \equiv s_{k+1}-s_{k}$, for $k \geq 1$. The solutions of the system (13) are given by

$$
\begin{aligned}
& R(t)=R\left(t_{k}^{+}\right) e^{-\omega_{1}\left(t-t_{k}\right)}, \quad t_{k}<t \leq t_{k+1} \\
& F(t)=F\left(t_{k}^{+}\right) e^{-\omega_{2}\left(t-s_{k}\right)}, \quad s_{k}<t \leq s_{k+1} .
\end{aligned}
$$

Now, in presence of the impulsive periodic orbits, we have a recurrence relation for the first equation of (17),

$$
R\left(t_{k}^{+}\right)=R\left(t_{k}^{-}\right)+\tilde{R}
$$

Then the drug concentration before the drug is taken is

$$
\begin{aligned}
R\left(t_{k}^{+}\right) & =\frac{\tilde{R}\left(1-e^{-k \omega_{1} \tau}\right)}{1-e^{-\omega_{1} \tau}}, \\
R\left(t_{k+1}^{-}\right) & =\frac{\tilde{R}\left(1-e^{-k \omega_{1} \tau}\right)}{1-e^{-\omega_{1} \tau}}+\tilde{R} \\
& =\frac{\tilde{R}\left(1-e^{-k \omega_{1} \tau}\right) e^{-\omega_{1} \tau}}{1-e^{-\omega_{1} \tau}} .
\end{aligned}
$$

Hence for the limiting cases, the drug concentration before and after dosing are

$$
\lim _{k \rightarrow \infty} R\left(t_{k}^{+}\right)=\frac{\tilde{R}}{1-e^{-\omega_{1} \tau}} \quad \text { and } \quad \lim _{k \rightarrow \infty} R\left(t_{k+1}^{-}\right)=\frac{\tilde{R} e^{-\omega_{1} \tau}}{1-e^{-\omega_{1} \tau}} .
$$

The similar arguments are valid for the fusion inhibitor $F(t)$ from (16) and (18).

Hence, the drugs impulsive periodic orbits for both drugs satisfy

$$
\frac{\tilde{R} e^{-t \tau}}{1-e^{-t \tau}} \leq R^{*} \leq \frac{\tilde{R}}{1-e^{-t \tau}}, \quad \text { and } \quad \frac{\tilde{F} e^{-t \sigma}}{1-e^{-t \sigma}} \leq F^{*} \leq \frac{\tilde{F}}{1-e^{-t \sigma}}
$$

where $t_{k}<t<t_{k+1}, \quad s_{k}<t<s_{k+1}$ with end points

$R\left(t_{k}^{+}\right)=\frac{\tilde{R}}{1-e^{-t \tau}}, \quad R\left(t_{k+1}^{-}\right)=\frac{\tilde{R} e^{-t \tau}}{1-e^{-t \tau}}, \quad$ and $\quad F\left(t_{k}^{+}\right)=\frac{\tilde{F}}{1-e^{-t \sigma}}, \quad F\left(t_{k+1}^{-}\right)=\frac{\tilde{F} e^{-t \sigma}}{1-e^{-t \sigma}}$.

\subsection{Disease free periodic orbit}

The disease free orbit is

$$
\hat{E}^{D}=\left(0, \hat{A}_{E}^{D}, \hat{A}_{E}^{\prime}, 0,0,0,0, \hat{M}_{E}^{D}, 0,0,0, R^{*}, F^{*}\right),
$$


where $\hat{A}_{E}^{D}=\frac{p e_{E}\left(m_{2}+\mu_{E}\right)}{\left(m_{2}+\mu_{E}+m_{1} R^{*}\right) \mu_{E}}, \hat{A}_{E}^{\prime}=\frac{m_{1} p e_{E} R^{*}}{\left(m_{2}+\mu_{E}+m_{1} R^{*}\right) \mu_{E}}$, and $\hat{M}_{E}^{D}=\frac{q e_{E}}{\mu_{E}}$ and $\eta^{*}=\left(1-\frac{F^{*}}{I C_{50}+F^{*}}\right)$.

Linearization of the impulsive differential equation of the model at the disease free orbit $\hat{E}_{D}$, takes the following form

$$
\frac{d U^{D}}{d t}=\left(S_{\hat{E}^{D}}-T_{\hat{E}^{D}}\right) U^{D}
$$

where $U^{D}=\left[S_{1 B}, C_{1}, S_{1 A}, C_{2}, S_{2}, C_{3}, I, V\right]^{T}$, and

$$
S_{\hat{E}^{D}}=\left(\begin{array}{cccccccc}
0 & 0 & 0 & 0 & 0 & 0 & 0 & a \\
\beta_{1} \hat{A}_{E}^{D} & 0 & 0 & 0 & 0 & 0 & 0 & 0 \\
0 & b & 0 & 0 & 0 & 0 & 0 & 0 \\
0 & 0 & \beta_{2} \hat{A}_{E}^{D} & 0 & 0 & 0 & 0 & 0 \\
0 & 0 & 0 & c & 0 & 0 & 0 & 0 \\
0 & 0 & 0 & 0 & \left(1-\eta^{*}\right) \beta_{3} \hat{M}_{E}^{D} & 0 & 0 & 0 \\
0 & 0 & 0 & 0 & 0 & \nu & 0 & 0 \\
0 & 0 & 0 & 0 & 0 & 0 & n d_{I} & 0
\end{array}\right)
$$

and

$$
T_{\hat{E}^{D}}=\left(\begin{array}{cccccccc}
\left(\mu_{S}+\beta_{1} \hat{A}_{E}^{D}\right) & 0 & 0 & 0 & 0 & 0 & 0 & 0 \\
0 & d_{1} & 0 & 0 & 0 & 0 & 0 & 0 \\
0 & 0 & \left(\mu_{S}+\beta_{2} \hat{A}_{E}^{D}\right) & 0 & 0 & 0 & 0 & 0 \\
0 & 0 & 0 & d_{2} & 0 & 0 & 0 & 0 \\
0 & 0 & 0 & 0 & \left(\mu_{S}+\left(1-\eta^{*}\right) \beta_{3} \hat{M}_{E}^{D}\right) & 0 & 0 & 0 \\
0 & 0 & 0 & 0 & 0 & d_{3} & 0 & 0 \\
0 & 0 & 0 & 0 & 0 & 0 & d_{I} & 0 \\
0 & 0 & 0 & 0 & 0 & 0 & 0 & \mu_{V}
\end{array}\right) .
$$

Basic reproduction ratio in presence of drug, $R_{0}^{D}$, can be derived using the spectral radius of next generation matrix. To find $R_{0}^{D}$, we must find the largest eigenvalue of $S_{\hat{E}^{D}} T_{\hat{E}^{D}}^{-1}$. Thus, $R_{0}^{D}=\rho\left(S_{\hat{E}^{D}} T_{\hat{E}^{D}}^{-1}\right)$, where

$$
\begin{aligned}
R_{0}^{D}= & {\left[\frac{n \nu a b c \beta_{1} \beta_{2} \beta_{3} I C_{50} p^{2} q e_{E}^{3}\left(m_{2}+\mu_{E}\right)^{2}}{d_{1} d_{2} d_{3} \mu_{V}\left\{\left(m_{2}+\mu_{E}\right)\left(\mu_{S} \mu_{E}+\beta_{1} p e_{E}\right)+\mu_{S} \mu_{E} m_{1} R^{*}\right\}}\right.} \\
& \left.\times \frac{1}{\left\{\left(m_{2}+\mu_{E}\right)\left(\mu_{S} \mu_{E}+\beta_{2} p e_{E}\right)+\mu_{S} \mu_{E} m_{1} R^{*}\right\}\left\{\mu_{S} \mu_{E}\left(I C_{50}+F^{*}\right)+\beta_{3} q e_{E} I C_{50}\right\}}\right]^{\frac{1}{8}} .
\end{aligned}
$$




\subsection{Endemic periodic orbit}

The endemic periodic orbit is in the form

$E^{* D}\left(S_{1 B}^{*}{ }^{D}, A_{E}^{* D}, A_{E}^{* D}, C_{1}^{* D}, S_{1 A}^{*}{ }^{D}, C_{2}^{* D}, S_{2}^{* D}, M_{E}^{* D}, C_{3}^{* D}, I^{* D}, V^{* D}, R^{*}, F^{*}\right)$, where

$$
\begin{aligned}
V^{* D} & =\frac{n d_{I} I^{* D}}{\mu_{V}} \\
C_{3}^{* D} & =\frac{d_{I} I^{* D}}{\nu} \\
M_{E}^{* D} & =\frac{q e_{E} \nu-d_{3} d_{I} I^{* D}}{\mu_{E} \nu} \\
S_{2}^{* D} & =\frac{\mu_{E} d_{3} d_{I} I^{* D}}{\left(1-\eta^{*}\right) \beta_{3}\left(q e_{E} \nu-d_{3} d_{I} I^{* D}\right)} \\
C_{2}^{* D} & =\frac{d_{3} d_{I}}{c}\left[\frac{\mu_{S} \mu_{E}}{\left(1-\eta^{*}\right) \beta_{3}\left(q e_{E} \nu-d_{3} d_{I} I^{* D}\right)}+\frac{1}{\nu}\right] I^{* D} \\
A_{E}^{\prime * D} & =\frac{m_{1} R^{*} A_{E}^{* D}}{m_{2}+\mu_{E}}, \\
S_{1 B}^{* D} & =\frac{a n d_{I} I^{* D}}{\mu_{V}\left(\mu_{S}+\beta_{1} A_{E}^{* D}\right)}, \\
S_{1 A}^{* D} & =\frac{a b n d_{I} \beta_{1} A_{E}^{* D} I^{* D}}{d_{2}\left(\mu_{S}+\beta_{1} A_{E}^{* D}\right)\left(\mu_{S}+\beta_{2} A_{E}^{* D}\right)}, \\
I^{* D} & =\frac{\nu\left(W_{1}+W_{2} A_{E}^{* D}+W_{3}\left(A_{E}^{* D}\right)^{2}\right)}{d_{3} d_{I}\left(1-\eta^{*}\right) \beta_{3}\left(W_{4}+W_{5} A_{E}^{* D}+W_{6}\left(A_{E}^{* D}\right)^{2}\right)}
\end{aligned}
$$

where, $W_{1}=d_{2}^{2} d_{3} \mu_{S}^{2}\left\{q e_{E}\left(1-\eta^{*}\right) \beta_{3}+\mu_{S} \mu_{E}\right\}, \quad W_{2}=d_{2}^{2} d_{3} \mu_{S}\left(\beta_{1}+\beta_{2}\right)\left\{q e_{E}(1-\right.$ $\left.\left.\eta^{*}\right) \beta_{3}+\mu_{S} \mu_{E}\right\}, \quad W_{3}=d_{2}^{2} d_{3} \beta_{1} \beta_{2}\left\{q e_{E}\left(1-\eta^{*}\right) \beta_{3}+\mu_{S} \mu_{E}\right\}-\operatorname{abcn\nu qe}_{E}(1-$ $\left.\eta^{*}\right) \beta_{1} \beta_{2} \beta_{3}, \quad W_{4}=d_{2}^{2} d_{3} \mu_{S}^{2}, \quad W_{5}=d_{2}^{2} d_{3} \mu_{S}\left(\beta_{1}+\beta_{2}\right), \quad W_{6}=\beta_{1} \beta_{2}\left(d_{2}^{2} d_{3}-\right.$ abcn $)$.

The value of $A_{E}^{* D}$ can be found from the equation,

$$
\begin{aligned}
& M_{1}\left(A_{E}^{* D}\right)^{5}+M_{2}\left(A_{E}^{* D}\right)^{4}+M_{3}\left(A_{E}^{* D}\right)^{3} \\
& +M_{4}\left(A_{E}^{* D}\right)^{2}+M_{5} A_{E}^{* D}+M_{6}=0
\end{aligned}
$$


where,

$$
\begin{aligned}
M_{1}= & p e_{E} d_{2} d_{3} \mu_{S}^{4}>0 \\
M_{2}= & \mu_{E}\left(1+\frac{m_{1} R^{*}}{m_{2}+\mu_{E}}\right)\left\{\mu_{S}\left(\beta_{1}+\beta_{2}\right) W_{6}+\beta_{1} \beta_{2} W_{5}\right\}-p e_{E} \beta_{1} \beta_{2} W_{6} \\
M_{3}= & \mu_{E}\left(1+\frac{m_{1} R^{*}}{m_{2}+\mu_{E}}\right)\left\{\mu_{S}^{2} W_{6}+\beta_{1} \beta_{2} W_{4}+\mu_{S}\left(\beta_{1}+\beta_{2}\right) W_{5}\right\} \\
& +\frac{a n \nu \beta_{1}}{d_{2} d_{3} \mu_{V}\left(1-\eta^{*}\right) \beta_{3}}\left\{d_{2} \mu_{S} W_{3}+\beta_{2}\left(d_{2}+b \mu_{V}\right) W_{2}\right\}-p e_{E}\left\{\mu_{S}\left(\beta_{1}+\beta_{2}\right) W_{6}+\beta_{1} \beta_{2} W_{5}\right\}, \\
M_{4}= & \mu_{S} \mu_{E}\left(1+\frac{m_{1} R^{*}}{m_{2}+\mu_{E}}\right)\left\{\mu_{S} W_{5}+\left(\beta_{1}+\beta_{2}\right) W_{4}\right\} \\
& +\frac{a n \nu \beta_{1}}{d_{2} d_{3}\left(1-\eta^{*}\right) \beta_{3} \mu_{V}}\left\{d_{2} \mu_{S} W_{2}+\left(d_{2}+b \mu_{V}\right) \beta_{2} W_{1}\right\} \\
& -p e_{e}\left\{\mu_{S}^{2} W_{6}+\beta_{1} \beta_{2} W_{4}+\mu_{S}\left(\beta_{1}+\beta_{2}\right) W_{5}\right\}, \\
M_{5}= & \mu_{E}\left(1+\frac{m_{1} R^{*}}{m_{2}+\mu_{E}}\right) \mu_{S}^{2} W_{4}+\frac{a n \nu \mu_{S} \beta_{1} W_{1}}{d_{3}\left(1-\eta^{*}\right) \mu_{V} \beta_{3}}-p e_{E} \mu_{S}\left\{\left(\beta_{1}+\beta_{2}\right) W_{4}+\mu_{S} W_{5}\right\}, \\
M_{6}= & -\beta_{1} \beta_{2} \mu_{E}\left(1+\frac{m_{1} R^{*}}{m_{2}+\mu_{E}}\right) W_{6} .
\end{aligned}
$$

Here we have the following result regarding the existence and stability of the disease-free periodic orbit and the endemic periodic orbit: Equation (21) will have a unique positive root if

- $M_{i}<0$ for $i=2, \ldots, 6$

- $M_{i}>0$ for $i=2,3,4,5$ and $M_{6}<0$.

Remark: If $R_{0}^{D}>1$, then the endemic periodic orbit $E^{* D}$ exists, either stable or unstable. We will later test its stability for a suitable choice of drug dose and dosing interval in section (4.2.2).

\subsubsection{Global Stability in Presence of Drug:}

To study the global stability of the system $\hat{E}^{D}$ we assume $x=\left(S_{1 B}, C_{1}, S_{1 A}, C_{2}, S_{2}, C_{3}, I, V\right), x \geq 0$. We have to verify that $\frac{d x}{d t} \leq$ $(S-T) x$ at $\hat{E}_{D}$, where $S_{\hat{E}^{D}}$ and $T_{\hat{E}^{D}}$ are defined in previous section.

Let $u$ is a left eigenvector associated with the eigenvalue $R_{0}^{D}$ of the matrix $\left(T^{-1} S\right)_{\hat{E}^{D}}$, such that

$$
u T_{\hat{E}^{D}}^{-1} S_{\hat{E}^{D}}=R_{0}^{D} u
$$


where $u>0$ follows from the fact $R_{0}^{D}=\rho\left(S T^{-1}\right)_{\hat{E}^{D}}=\rho\left(T^{-1} S\right)_{\hat{E}^{D}}$.

Let us consider the Lyapunov function

$$
\mathbb{L}=u T_{\hat{E}^{D}}^{-1} x .
$$

Differentiation (23), we have

$$
\begin{aligned}
\mathbb{L}^{\prime} & =u T_{\hat{E}^{D}}^{-1} x^{\prime} \\
& \leq u T_{\hat{E}^{D}}^{-1}(S-T) x \\
& =u\left(R_{0}^{D}-1\right) x .
\end{aligned}
$$

Therefore, when $R_{0}^{D}<1, \mathbb{L}^{\prime}<0$ unless $x=0$ and $\mathbb{L}=0$ when $u x=0$.

This leads $S_{1 B}=C_{1}=S_{1 A}=C_{2}=S_{2}=C_{3}=I=V=0$ by noting the positive component of $u$ and $A_{E}={\hat{A_{E}}}^{D}, A_{E}^{\prime}={\hat{A_{E}^{\prime}}}^{D}, M_{E}=\hat{M}_{E}{ }^{D}$ when $R_{0}^{D}<1$.

Therefore the invariant set on which $\mathbb{L}=0$ contains a unique point, which is disease free periodic orbit $\hat{E}^{D}$ at which the system is globally stable, and $E^{* D}$ does not exist.

\subsubsection{Successful and unsuccessful treatment regimens:}

We now examine the effect of the drug dosage over the system.

Theorem 2. Keeping fusion inhibitor at a constant dose, we consider the following cases:

- If $\tilde{R}>R_{1}$, the disease free periodic orbit $\hat{E}^{D}$ is globally stable and $E^{* D}$ does not exist.

- When $0 \leq \tilde{R}<R_{2}, \hat{E}^{D}$ is unstable and $E^{* D}$ exist,

where $R_{1}=\frac{\chi}{\phi_{1}}$ and $R_{2}=\frac{\chi}{\phi_{2}}$, and $\phi_{1}=\frac{e^{-\omega_{1} \tau}}{1-e^{-\omega_{1} \tau}}, \phi_{2}=\frac{1}{1-e^{-\omega_{1} \tau}} \cdot \chi$ denotes the drug level of $R^{*}$ when $R_{0}^{D}=1$ and is define as

$$
\xi_{1}\left(R^{*}\right)^{2}+\xi_{2} R^{*}+\xi_{3}=0
$$

where

$$
\begin{aligned}
\xi_{1}= & \left(\mu_{S} \mu_{E} m_{1}\right)^{2}, \\
\xi_{2}= & \mu_{S} \mu_{E} m_{1}\left(m_{2}+\mu_{E}\right)\left\{2 \mu_{S} \mu_{E}+\left(\beta_{1}+\beta_{2}\right) p e_{E}\right\} \\
\xi_{3}= & \left(m_{2}+\mu_{E}\right)^{2}\left[\left(\mu_{S} \mu_{E}+\beta_{1} p e_{E}\right)\left(\mu_{S} \mu_{E}+\beta_{2} p e_{E}\right)\right. \\
& \left.-\frac{n \nu a b c \beta_{1} \beta_{2} \beta_{3} p^{2} q e_{E}^{3} I C_{50}}{d_{1} d_{2} d_{3} \mu_{V}\left\{\mu_{S} \mu_{E}\left(I C_{50}+F^{*}\right)+q e_{E} \beta_{3} I C_{50}\right\}}\right] .
\end{aligned}
$$


Proof. We have

$$
\tilde{R} \phi_{1} \leq R^{*} \leq \tilde{R} \phi_{2}
$$

Now, if $\tilde{R}>R_{1}$, then

$$
\begin{aligned}
\tilde{R} & >\frac{\chi\left(1-e^{-\omega_{1} \tau}\right)}{e^{-\omega_{1} \tau}} \\
& \Rightarrow \frac{e^{-\omega_{1} \tau} \tilde{R}}{1-e^{-\omega_{1} \tau}}>\chi \\
& \Rightarrow \chi<R^{*}
\end{aligned}
$$

Thus,

$$
\begin{aligned}
\xi_{1}\left(R^{*}\right)^{2}+\xi_{2} R^{*}+\xi_{3} & >0 \\
\Rightarrow R_{0}^{D} & <1 .
\end{aligned}
$$

It follows that $\hat{E}^{D}$ is globally stable and $E^{* D}$ does not exist. If $\tilde{R}<R_{2}$, then

$$
\begin{aligned}
\tilde{R} & <\chi\left(1-e^{-\omega_{1} \tau}\right) \\
\Rightarrow \frac{\tilde{R}}{1-e^{-\omega_{1} \tau}} & <\chi \\
\Rightarrow R^{*} & <\chi .
\end{aligned}
$$

Then,

$$
\begin{aligned}
\xi_{1}\left(R^{*}\right)^{2}+\xi_{2} R^{*}+\xi_{3} & <0 \\
\Rightarrow R_{0}^{D} & >1 .
\end{aligned}
$$

It follows that $\hat{E}^{D}$ is unstable and $E^{* D}$ exist.

Also when $R_{0}^{D}<1$, we have the threshold value of the drug dose $R_{1}$ such that,

$$
\begin{aligned}
\tilde{R} & >R_{1} \\
\Rightarrow \tilde{R} & >\frac{\chi\left(1-e^{-\omega_{1} \tau}\right)}{e^{-\omega_{1} \tau}} \\
\Rightarrow \tau & <\frac{1}{\omega_{1}} \ln \left(1+\frac{\tilde{R}}{\chi}\right) \equiv \tau_{1} .
\end{aligned}
$$


If $R_{0}^{D}>1$, we have found the threshold value of the drug dose, $R_{2}$, such that

$$
\begin{aligned}
\tilde{R} & <R_{2} \\
\Rightarrow \tilde{R} & <\chi\left(1-e^{-\omega_{1} \tau}\right) \\
\Rightarrow \tau & >-\frac{1}{\omega_{1}} \ln \left(1-\frac{\tilde{R}}{\chi}\right) \equiv \tau_{2} .
\end{aligned}
$$

Keeping the fusion inhibitor fixed, the disease free periodic orbit will be stable if the drug regimen satisfies the conditions $\tilde{R}>R_{1}$, i.e. when the drug dose is sufficiently high. If $\tilde{R}<R_{2}$, the infection persists and reaches to an endemic state. Thus we can conclude that if the dosing interval $\tau$ satisfy the relation $0 \leq \tau<\tau_{1}$ for a fixed dosage, then the disease free periodic orbit will be stable. If $\tau>\tau_{2}$ then the endemic periodic orbit will exist.

Theorem 3. Let $F_{1}=\frac{\Pi}{\psi_{1}}, \quad F_{2}=\frac{\Pi}{\psi_{2}}$, where $\psi_{1}=\frac{e^{-\omega_{2} \sigma}}{1-e^{-\omega_{2} \sigma}}$ and $\psi_{2}=$ $\frac{1}{1-e^{-\omega_{2} \sigma}}$, and

$$
\begin{aligned}
\Pi= & \frac{I C_{50}\left[n \nu a b c p^{2} q e_{E}^{3} \beta_{1} \beta_{2} \beta_{3}\left(m_{2}+\mu_{E}\right)^{2}-d_{1} d_{2} d_{3} \mu_{V}\left\{\left(m_{2}+\mu_{E}\right)\left(\mu_{S} \mu_{E}+\beta_{1} p e_{E}\right)+\mu_{S} \mu_{E} m_{1} R^{*}\right\}\right.}{d_{1} d_{2} d_{3} \mu_{V} \mu_{E} \mu_{S}\left\{\left(m_{2}+\mu_{E}\right)\left(\mu_{S} \mu_{E}+\beta_{1} p e_{E}\right)+\mu_{S} \mu_{E} m_{1} R^{*}\right\}} \\
& \times \frac{\left.\left\{\left(m_{2}+\mu_{E}\right)\left(\mu_{S} \mu_{E}+\beta_{2} p e_{E}\right)+\mu_{S} \mu_{E} m_{1} R^{*}\right\}\left(\mu_{S} \mu_{E}+\beta_{3} q e_{E}\right)\right]}{\left\{\left(m_{2}+\mu_{E}\right)\left(\mu_{S} \mu_{E}+\beta_{2} p e_{E}\right)+\mu_{S} \mu_{E} m_{1} R^{*}\right\}} .
\end{aligned}
$$

Keeping the HCQ drug dose constant, we consider the following cases

- If $\tilde{F}>F_{1}, \hat{E}^{D}$ is globally stable and $E^{* D}$ does not exist.

- If the dosage satisfy $0 \leq \tilde{F}<F_{2}, \hat{E}^{D}$ is unstable and $E^{* D}$ exists.

Proof. We have $\tilde{F} \psi_{1} \leq F^{*} \leq \tilde{F} \psi_{2}$. If $\tilde{F}>F_{1}$, then

$$
\begin{array}{ll}
\tilde{F}> & \frac{\Pi\left(1-e^{-\omega_{2} \sigma}\right)}{e^{-\omega_{2} \sigma}} \\
\Rightarrow & \frac{e^{-\omega_{2} \sigma} \tilde{F}}{1-e^{-\omega_{2} \sigma}}>\Pi \\
\Rightarrow & \Pi<F^{*} \\
\Rightarrow & R_{0}^{D}<1 .
\end{array}
$$


It follows that $\hat{E}^{D}$ is globally stable and $E^{* D}$ does not exist. Similarly, if $\tilde{F}<F_{2}$, then

$$
\begin{array}{ll}
\tilde{F}< & \Pi\left(1-e^{-\omega_{2} \sigma}\right) \\
\Rightarrow & \frac{\tilde{F}}{1-e^{-\omega_{2} \sigma}}<\Pi \\
\Rightarrow \quad & F^{*}<\Pi \\
\Rightarrow & R_{0}^{D}>1 .
\end{array}
$$

It follows that $\hat{E}^{D}$ is unstable and $E^{* D}$ exists.

When $R_{0}^{D}<1$, we have a threshold value of the drug dose $F_{1}$, such that

$$
\begin{aligned}
\tilde{F} & >F_{1}, \\
\Rightarrow \sigma & <\frac{1}{\omega_{2}} \ln \left(1+\frac{\tilde{F}}{\Pi}\right) \equiv \sigma_{1} .
\end{aligned}
$$

When $R_{0}^{D}>1$, we have threshold value of the drug dose $F_{2}$, such that

$$
\begin{aligned}
\tilde{F} & <F_{2} \\
\Rightarrow \sigma & >-\frac{1}{\omega_{2}} \ln \left(1-\frac{\tilde{F}}{\Pi}\right) \equiv \sigma_{2} .
\end{aligned}
$$

Hence, we can conclude that keeping the HCQ dosages fixed, the disease free periodic orbit will be stable if the drug regimen satisfies the condition $\tilde{F}>F_{1}$, for a fixed dosing interval, i.e. drug dosage is sufficiently high or $0 \leq$ $\sigma<\sigma_{1}$ (when the dosage is constant). Also if $\tilde{F}<F_{2}$, the infection persists and reaches its endemic periodic orbit. If the dosage is fixed and $\sigma>\sigma_{2}$, the endemic periodic orbit will exist in presence of the drug. The prediction is impossible if the dosing interval satisfies the relation $\sigma_{1}<\sigma<\sigma_{2}$ or the dosage interval $F_{2}<\tilde{F}<F_{1}$, because $R_{0}^{D}$ fluctuates around one.

Remark 1: Fig.7 and Fig.8 illustrate the outcome for the drug regimen $\tilde{F}=0.04>F_{1}, \tilde{R}=0.06>R_{1}$ with dosing interval $\tau=\sigma=0.5$. Here we consider two cases, first one is treatment starts after 10 days of detection and another one is immediate after detection. We observed that if the treatment start after immediate detection then the disease will be controlled within 10-12 days. But if we start the treatment after 10 days of the detection, then it will take minimum 30 to 35 days to control the disease. From these 

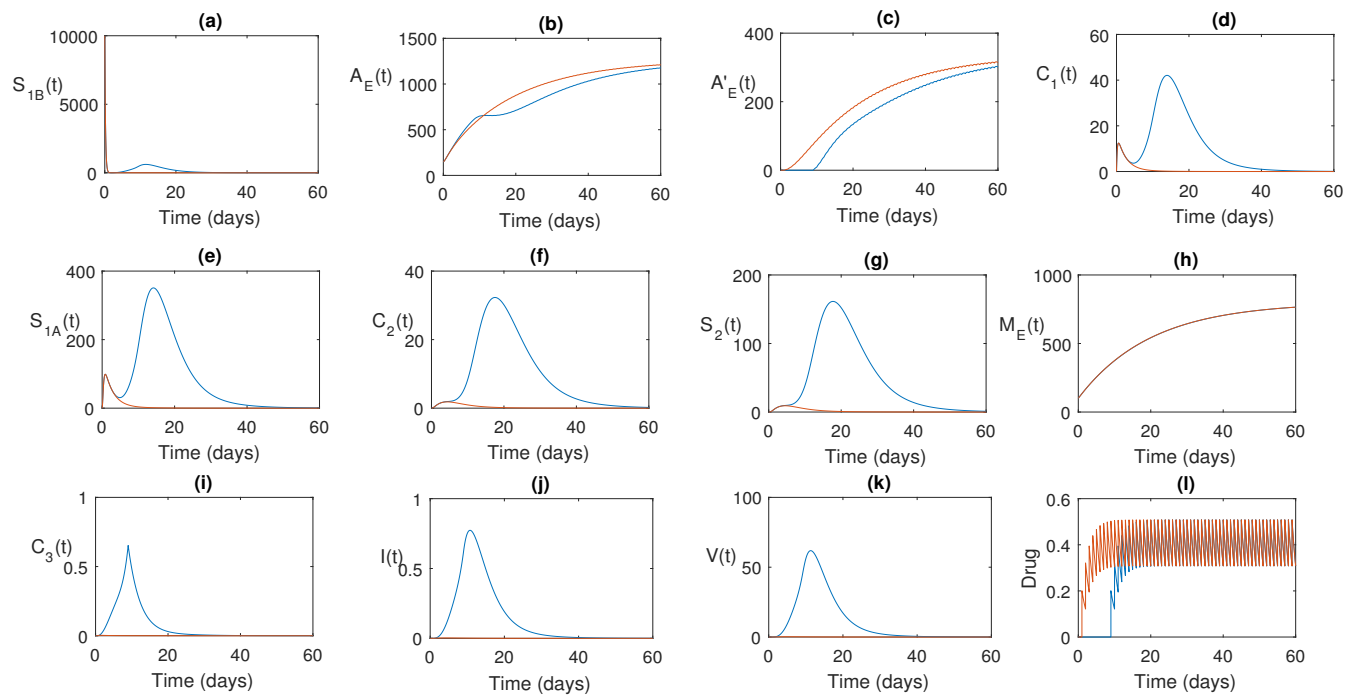

Figure 7: Blue line: treatment starts after 10 days, Red line: treatment starts immediate after detection with $\tau=0.5, \sigma=0.5$ and $\tilde{F}=0.04, \tilde{R}=$ 0.06 .
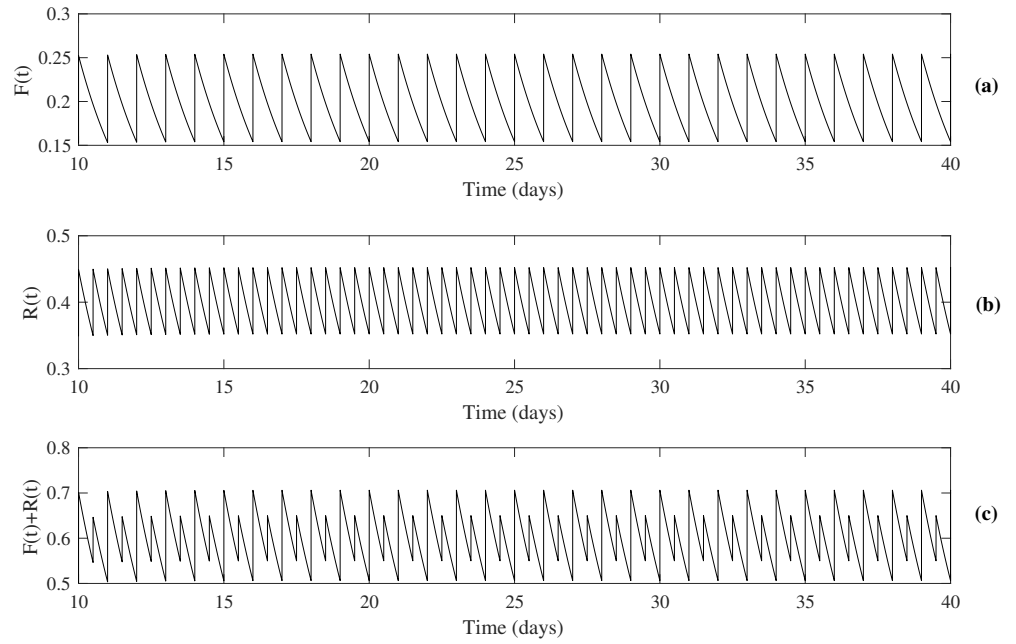

Figure 8: Time dependent changes of concentration of drugs with $\tilde{R}=0.06$ and $\tilde{F}=0.04$. 

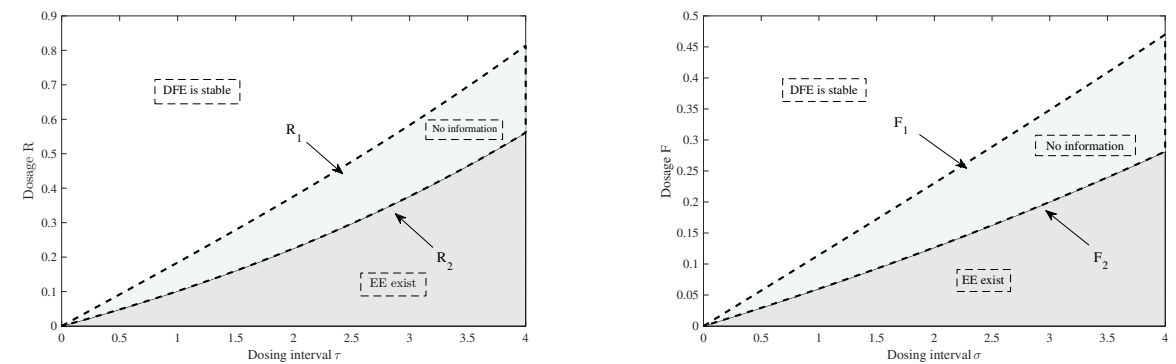

Figure 9: Left panel: Regions of stability and unstability for dosage $\tilde{R}$ and dosing interval $\tau$. Right panel: Region of stability and unstability for the dosage $\tilde{F}$ and dosing interval $\sigma$.

observations it revels that early treatment must be needed to control the disease and no such side effect would be occurred.

Remark 2: We have plotted the region of stability in Fig.9A and Fig.9B for the parameter set in Table. 1. These figures show that if $\tilde{R}$ (or $\tilde{F}$ ) is sufficiently small and $\tau$ (or $\sigma$ ) suitably large, then $\hat{E}^{D}$ is guaranteed to be occurred as stable in nature and $E^{* D}$ does not exist. If $\tilde{R}$ (or $\tilde{F}$ ) is sufficiently large or $\tau$ (or $\sigma$ ) is suitably small, then $\hat{E}^{D}$ is assured to be occurred as unstable in nature and $E^{* D}$ does exist. Also it is evident from this figure that the safe region of stability for the $\operatorname{drug} \tilde{F}$ is much wider than that of $\tilde{R}$. It means $\tilde{F}$ is more sensitive and effective compared with the drug $\tilde{R}$.

Remark 3: In Fig.10, we have plotted Poincaré section for the equation (21) and for a safe dose and dosing interval. We choose values $\tilde{R}=$ 0.06, $\tau=0.5$ and $\tilde{F}=0.04 \sigma=0.5$ from the upper stable region of Fig.9A and Fig.9B. Other parameter values are taken from the Table.1. This figure reveals that the points lie on a particular pattern occupying a subset of phase space. Trajectories intersecting the plane somewhere on this pattern clearly form an attractor for this dynamics. This attractor is a limit cycle and the system is stable.

Remark 4: Further, we draw maximum Lyapunov exponent of the system (12)-(16) for $\tilde{R}=0.05565, \tau=0.5$ and $\tilde{F}=0.03376 \sigma=0.5$ (Fig.11), which shows that the system becomes stable from the chaotic dynamics after 1.5 weeks or 10-11 days. Other parameter values are chosen from the Table.1.

Remark 5: We have explored variation in some uncertain parameters. Fig. 12 gives the graph of $R_{0}$ (Left Panel) and $R_{0}^{D}$ (Right Panel) as a function 


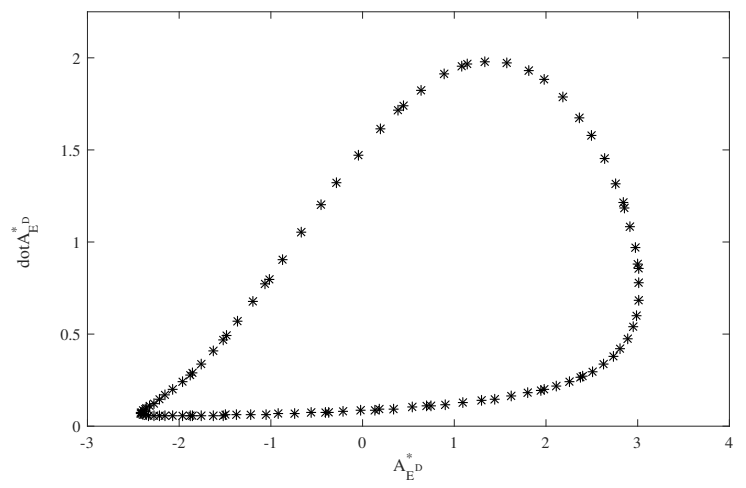

Figure 10: Poincaré section for the set of parameter values given in the Table. 1

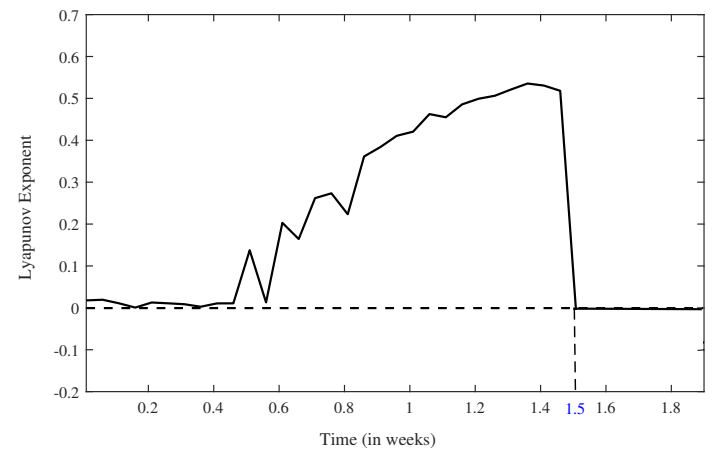

Figure 11: Figure shows maximum Lyapunov exponent of the system (12)(16) for $\tilde{R}=0.05565, \tau=0.5$ and $\tilde{F}=0.03376, \sigma=0.5$. Other parameter values are chosen from the Table.1. 

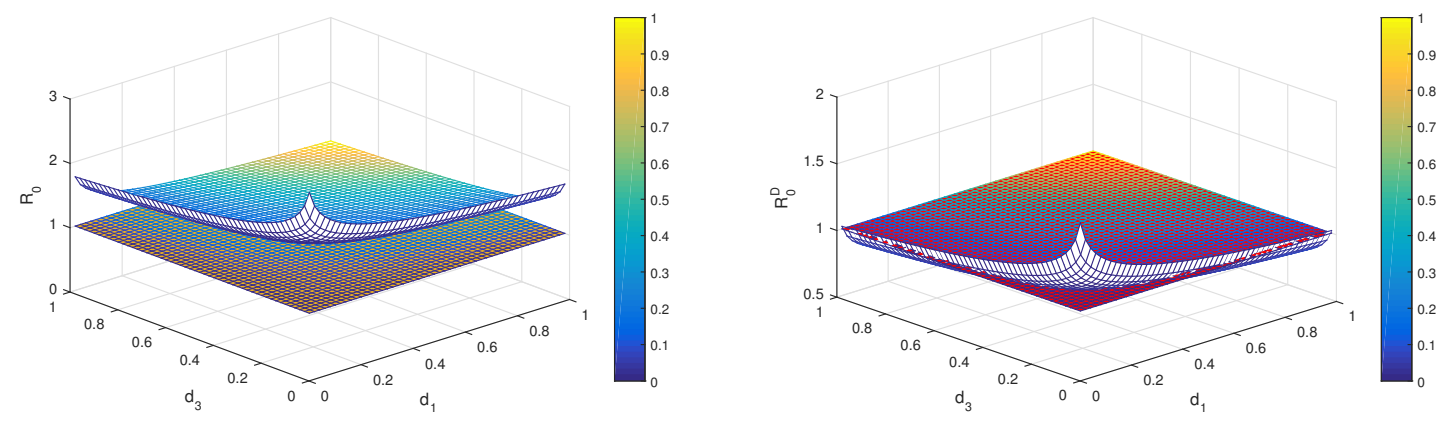

Figure 12: Left Panel: The graph of $R_{0}$ as a function of $d_{1}$ and $d_{3}$. Right Panel: The graph of $R_{0}^{D}$ as a function of $d_{1}$ and $d_{3}$.
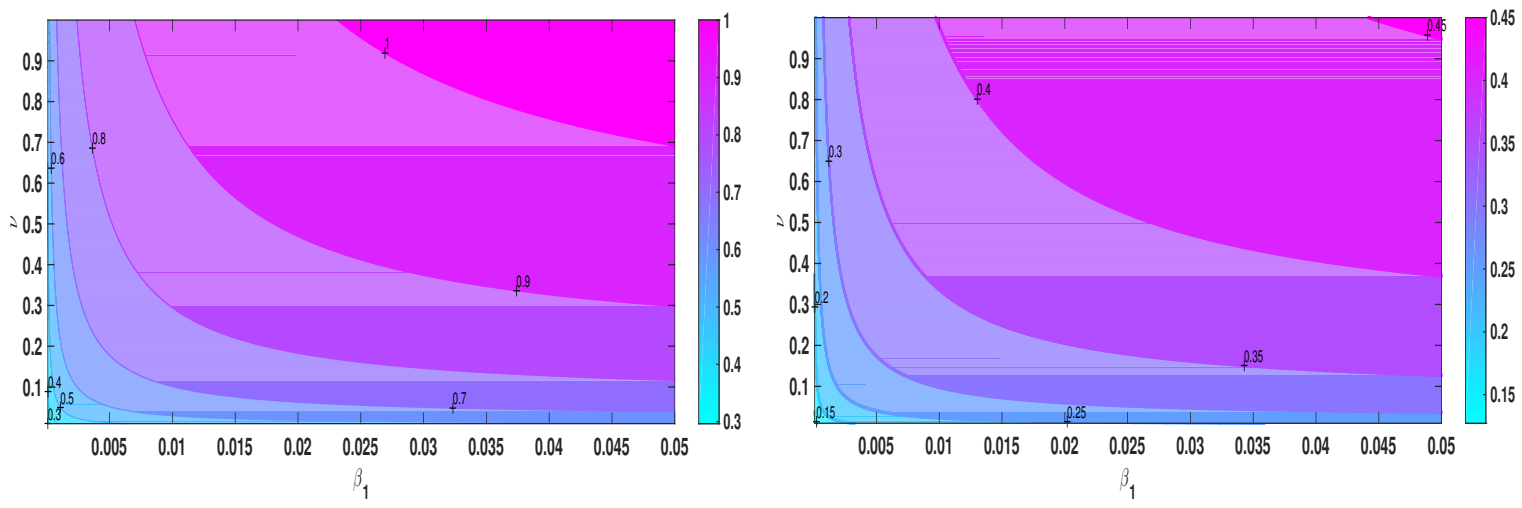

Figure 13: Left Panel: $R_{0}$ as a function of $\beta_{1}$ and $\nu$. Right Panel: $R_{0}^{D}$ as a function of $\beta_{1}$ and $\nu$. 

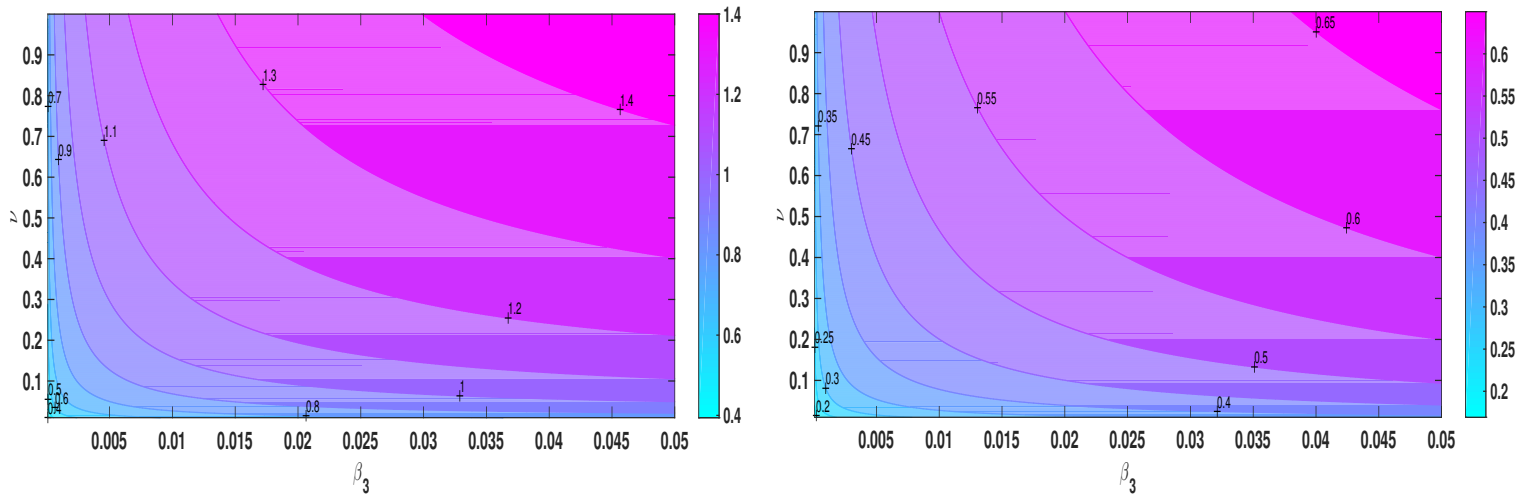

Figure 14: Left Panel: $R_{0}$ as a function of $\beta_{3}$ and $\nu$. Right Panel: $R_{0}^{D}$ as a function of $\beta_{3}$ and $\nu$.

of $d_{1}$ and $d_{3}$. These illustrate the change of the threshold parameter $R_{0}$ and $R_{0}^{D}$ as $d_{1}$ and $d_{3}$ vary. Clearly, if $d_{1}$ and $d_{3}$ are sufficiently large, then the basic reproductive number gives lower values when drug is implemented in the system. Again, we plot $R_{0}\left(\beta_{1}, \nu\right)$ and $R_{0}^{D}\left(\beta_{1}, \nu\right)$ as a function of $\beta_{1}$ and $\nu$, and $R_{0}\left(\beta_{3}, \nu\right)$ and $R_{0}^{D}\left(\beta_{3}, \nu\right)$ as a function of $\beta_{3}$ and $\nu$ in Fig. 13 and Fig.14, respectively. Comparing these two, we come into the conclusion that as soon as the values of $\beta_{1}, \beta_{3}$ and $\nu$ get smaller, the value of basic reproductive number blow down to less than 1 . System (1)-(3) fits better compare to system (12)-(16) in that mathematical stand point.

\section{Discussion and conclusion}

The spread of coronavirus disease 2019 (COVID-19) is increasing rapidly and scientist are in different field in the Globe striving to discover the drugs for its effective treatment. We have formulated a basic mathematical model of COVID -19/ SARS-CoV-2 to study the cellular changing aspect of the disease with combination of drug therapy Hydroxychloroquine (HCQ) and lipopeptide EK1C4 to design the probable control of the pandemic. We have incorporated impulsive differential equations model to preview the drug dynamics with a perfect adherence therapeutic strategies for both drugs which plays a pivotal role in continuing clinical trials. It is revealed that the mechanism of drug interaction is not instantaneous. To overcome this, using impulsive differential equation, it has been shown a reasonable approximation to the agreement of drug intake. 
In this research work with few constraints we consider the growth rate of $S_{1 A}$ is directly proportional to $C_{1}$ (the combination of S1B and ACE2 receptor) Also, the bonding force between $S_{1 A}$ and $A_{E}\left(\beta_{2}\right)$ depends on $C_{1}$. We further assume that the growth rate of $S_{2}$ is directly proportional to $C_{2}$ and the bonding force $\beta_{3}$ between $S_{2}-M_{E}$ is depends on $C_{2}$. Finally, we assume that the production of newly infected cells which is directly proportional to $C_{3}$ as in reality it is more complicated.

In this article, we have studied the effective measure of equilibrium steady states of the system for without drug and establish the conditions under which the equilibriums switch their stabilities from stable to unstable. We have restrained the threshold reproduction number that emphases parameter make it utmost or less sensitive in to the system. It is also perceived that if the basic reproduction number, $R_{0}$ is less than unity, then the disease free equilibrium (DFE) preserve globally stable but the system switches its stability whenever the value of $R_{0}$ exceeds unity. It should be mentioned here that due to critical situation $R_{0}$ can be quite high. However, appropriate drug strategies can control the virus (Fig.7, Fig.8) but without treatment the system become a chaotic in nature (Fig.3).

During the treatment period of a Covid19 patient, it is also vital to provide an effective drug regimen. Not only it is important that the drugs have a high efficacy, but it is also significant that it will be beneficial for both their mental and physical states. We have analysed the model with drugs, Hydroxychloroquine (HCQ) followed by lipopeptide EK1C4. The HCQ drug molecules are less toxic and inhibit viral infection in cell culture. Fusion inhibitor lipopeptide EK1C4 act in a different way to control the disease. It's preventing the virions from entry into the target cell by bonding with viral surface proteins. Numbers of fusion inhibitor molecules prevent virions from entering the cell is dependent on the number of trimmers on the cell surface. It has been observed that for a proper choice of drug dose $(R=0.05565, F=0.03376)$ with a proper dosing interval $(\tau=\sigma=0.5$, that is twice a day) the system becomes stable from chaotic dynamics after 11 days (Fig.11).

Insight of the probable treatment schedule, we have demonstrated the effects of taking drug dose and dosing interval during the active infection period. It has a great impact to determine the dose regime for a finite time (short time) treatment policy. Considering from analytical view point of theorem 4.1 and 4.2, we have two categories of threshold values. For Hydroxychloroquine (HCQ), the threshold values are $R_{1}=0.04818, R_{2}=$ 0.05565 and for lipopeptide $F_{1}=0.02958, F_{2}=0.02793$. If we consider the drug dosage $\tilde{R}>R_{1}=0.04818$ and $\tilde{F}>F_{1}=0.04818$, then the system 
attains its safe region and the disease can be controlled. We have also observed if $\tilde{R}<R_{2}=0.0436$ and $\tilde{F}<F_{1}=0.02793$, the system moves to unsafe region. From these findings we can predict that lipopeptide EK1C4 is more effective compare to Hydroxychloroquine.

Due to lack of sufficient primary data, we have chosen our parameter values to see the dynamical behaviours namely asymptotic stability, the threshold reproduction number that tells which parameter is most or less sensitive to the system. Other hand, the proposed treatment regime using Hydroxychloroquine, lipopeptide EK1C4 is based on hypothetical value of model parameters, but the result can be proposed for future clinical trials. It is also possible to determine the therapy schedule for drugs (chloroquine, arbidol, Remdesivir, favipiravir Tocilizumab, Duvelisib etc.) using this analytical and numerical techniques. Moreover, if proper data are obtained and a particular situation can be modelled, then we will be able to make these proposed results more applicable and biologically reasonable.

Thus in a nutshell, the proposed mathematical model successfully described the dynamics of COVID -19/SARS-CoV-2 in cellular level. Furthermore, impulsive differential equations are a useful mathematical tool for elucidating insights into regular drug dosing. Results from analytical as well as numerical findings recommend that the initial stage of detection of COVID-19 patients will be recovered within 10 to 12 days soon after using the combination of drug. But if the treatment starts after 10 days of infection/detection, it may take 30-35 days to recover. Such delay of treatment causes a high mortality to the patient due to lower immunity and massive proliferation of viral load which ultimately affect the lungs. So early detection and instant treatment is highly recommendable for controlling the COVID-19 patients.

ACKNOWLEDGEMENT: Priti Kumar Roy is supported by the Indo-Russian joint research project INT/RUS/RFBR/P-306 organized by DST-RFBR 2018. Evgenii N. Khailov is funded by Russian Foundation for Basic Research according to the research project 18-51-45003 IND a.

The authors have no conflict of interest.

\section{References}

[1] Zhou, P. et al. (2020) A pneumonia outbreak associated with a new coronavirus of probable bat origin. Nature. Published online February 3,2020 , 
[2] Menachery, V.D. et al. (2016) SARS-like WIV1-CoV poised for human emergence. Proc. Natl. Acad. Sci. U. S. A., 113, 3048--3053.

[3] Belouzard, S. et al. (2009) Activation of the SARS coronavirus spike protein via sequential proteolytic cleavage at two distinct sites, Proc. Natl. Acad. Sci. U. S. A., 106, 5871--5876.

[4] Martin J. V., Eric B., Suzanne B., Bobbie R. E., Pierre E. R., Thomas G. K., Nabil G. S., Stuart T. N. (2020) Chloroquine is a potent inhibitor of SARS coronavirus infection andspread, Virology Journal, 1-10.

[5] Shuai X., Meiqin L., Chao W., Wei X., Qiaoshuai L., Siliang F., Feifei Q., Linlin B., Lanying D., Shuwen L., Chuan Q., Fei S., Zhengli S., Yun Z., Shibo J., Lu L., (2020) Inhibition of SARS-CoV-2 (previously 2019$\mathrm{nCoV}$ ) infection by a highly potent pan-coronavirus fusion inhibitor targeting its spike protein that harbors a high capacity to mediate membrane fusion, Cell research, 343-355.

[6] Savarino, A. et al. (2006) New insights into the antiviral effects of chloroquine, Lancet Infect. Dis., 6, 67--69.

[7] Jia Liu, Ruiyuan Cao, Mingyue Xu, Xi Wang, Huanyu Zhang, Hengrui $\mathrm{Hu}$, Yufeng Li, Zhihong Hu, Wu Zhong and Manli Wang, (2020) Hydroxychloroquine, a less toxic derivative of chloroquine, is effective in inhibiting SARS-CoV-2 infection in vitro, Cell Discovery, 6:16.

[8] Van, B. S., De Graaf, M., Lauber, C., Bestebroer, T. M., Raj, V. S., Zaki, A. M., Osterhaus, A. D., Haagmans, B. L., Gorbalenya, A. E, Snijder E. J., Fouchier, R. A. (2012) Genomic characterization of a newly discovered coronavirus associated with acute respiratory distress syndrome in humans, mBio., 3(6):e00473-12.

[9] Perlman S, Dandekar AA. (2005) Immunopathogenesis of coronavirus infections: implications for SARS, Nat Rev Immunol., 5, 917-927.

[10] ,World Health Organization (2020). WHO Director-General's opening remarks at the media briefing on COVID-19-11 March 2020. Geneva, Switzerland.

[11] Perlman, S., Netland, J. (2009) Coronaviruses post-SARS: update on replication and pathogenesis, Nat Rev Microbiol., 7, 439-450.

[12] Peiris, J. S., Guan, Y., Yuen, K. Y. (2004) Severe acute respiratory syndrome., Nat Med., 10, 88--97. 
[13] Rudragouda C., Zhao, J., Perlman, S. (2014) T cell-mediated immune response to respiratory coronaviruses, Immunol Res, 59, 118--128.

[14] Wan, Y., Shang, J., Graham, R., Baric, R.S. and Li, F., (2020) Receptor recognition by the novel coronavirus from Wuhan: an analysis, based on decade-long structural studies of SARS coronavirus., Journal of virology, $94(7)$.

[15] Hsueh-Ling J. O., Samuel K. G., Antonio B., Yee-Joo T., (2012) Understanding the $\mathrm{T}$ cell immune response in SARS coronavirus infection, Emerging Microbes and Infections, 1, e23; doi:10.1038/emi.2012.26.

[16] Li, G. and De Clercq, E., (2020) Therapeutic options for the 2019 novel coronavirus (2019-nCoV).

[17] Chan-Yeung, M., Xu, R. H. (2003) SARS: epidemiology., Respirology,8, $9--14$.

[18] Song, Z., Xu, Y., Bao, L., Zhang, L., Yu, P., Qu, Y., Zhu, H.,Wenjie Zhao, Yunlin Han, Chuan Qin,(2019) From SARS to MERS, Thrusting Coronaviruses into the Spotlight, Viruses, 11, 59.

[19] Meyerholz, D.K., Lambertz, A.M., McCray, P.J., (2016) Dipeptidyl Peptidase 4 Distribution in the Human Respiratory Tract: Implications for the Middle East Respiratory Syndrome., Am. J. Pathol., 186, 78-86 .

[20] Widagdo, W., Raj, V.S., Schipper, D., Kolijn, K., van Leenders, G., Bosch, B.J., Bensaid, A., Segales, J., Baumgartner, W., Osterhaus, A., (2016) Differential Expression of the Middle East Respiratory Syndrome Coronavirus Receptor in the Upper Respiratory Tracts of Humans and Dromedary Camels., J. Virol., 90, 4838--4842.

[21] Chu, K. H., Tsang, W.K., Tang, C.S., Lam, M. F., Lai, F. M., To, K. F., Fung, K. S., Tang, H. L., Yan, W. W., Chan, H. W. (2005) Acute renal impairment in coronavirus-associated severe acute respiratory syndrome.. Kidney Int., 67, 698--705.

[22] Saad, M., Omrani, A. S., Baig, K., Bahloul, A., Elzein, F., Matin, M. A., Selim, M. A., Al, M. M., Al, N. D., Al, A. A. (2014) Clinical aspects and outcomes of 70 patients with Middle East respiratory syndrome coronavirus infection: A single-center experience in Saudi Arabia., Int. J. Infect. Dis., 29, 301--306. 
[23] Lindi M. W., Martin A. N., (2000) Adherence and drug resistance: predictions for therapy outcome, Proc. R. Soc. Lond. B, 267, 835-843.

[24] Korobeinikov, A. (2004) Global properties of basic virus dynamics models, Bull Math Biol, 66(4), 879-883.

[25] Hethcote, H. W.,(2000) The mathematics of infectious disease, SIAM Rev., 42 (4), 599-653.

[26] Xia, S., Liu, M., Wang, C., Xu, W., Lan, Q., Feng, S., Qi, F., Bao, L., Du, L., Liu, S., Qin, C., Sun, F., Shi, Z., Zhu, Y., Jiang, S. Lu, L. (2020) Inhibition of SARS-CoV-2 (previously 2019-nCoV) infection by a highly potent pan-coronavirus fusion inhibitor targeting its spike protein that harbors a high capacity to mediate membrane fusion, Cell Research, 30 343--355.

[27] Wrapp, D., Wang, N., Corbett, K. S., Goldsmith, J. A., Hsieh, C. L., Abiona, O., Graham, B. S., McLellan, J. S., Cryo, E. M. (2020) structure of the 2019-nCoV spike in the prefusion conformation. Science, 367, $1260-1263$.

[28] Rabi, F. A., Mazhar S. Al Zoubi, Ghena A. Kasasbeh, Dunia M. Salameh, Amjad D. Al-Nasser (2020) SARS-CoV-2 and Coronavirus Disease 2019: What We Know So Far, Pathogens, 9, 231.

[29] Walls, A., Park, Y. J., Tortorici, M. A., Wall, A., McGuire, A. T., Veesler, D. (2020) Structure, Function, and Antigenicity of the SARSCoV-2 Spike Glycoprotein, Cell, 180, 281--292.

[30] Guillon, P., Monique, C., V'eronique. S., Rivain, J. G., Chou, C. F., Nathalie R.C., Jacques L. P.(2008) Inhibition of the interaction between the SARS-CoV Spike protein and its cellular receptor by anti-histoblood group antibodies, Glycobiology, 18, 1085--1093.

[31] Lou, J., Smith, R. J. (2011) Modelling the effects of adherence to the HIV fusion inhibitor enfuvirtide, J. Theor. Biol, 268, 1--13.

[32] Chatterjee, A. N., Roy, P. K.(2012) Anti-viral drug treatment along with immune activator IL-2: A control-based mathematical approach for HIV infection, Int. J. Control, 85(2), 220--237.

[33] Roy, P. K., Chatterjee, A. N., Li, X-Z (2016) The effect of vaccination to dendritic cell and immune cell interaction in HIV disease progression, Int J Biomath, 9(1):1650005, 2016. 